Article

\title{
Multiobjective Combination Optimization of an Impeller and Diffuser in a Reversible Axial-Flow Pump Based on a Two-Layer Artificial Neural Network
}

\author{
Fan Meng ${ }^{1}$, Yanjun $\mathrm{Li}^{1}{ }^{1, *}$, Shouqi Yuan ${ }^{1}$, Wenjie Wang ${ }^{1}{ }^{1}$, Yunhao Zheng ${ }^{1}$ and \\ Majeed Koranteng Osman 1,2 (D) \\ 1 Research Center of Fluid Machinery Engineering and Technology, Jiangsu University, Zhenjiang 212013, \\ China; mengf2456@126.com (F.M.); shouqiy@ujs.edu.cn (S.Y.); wenjiewang@ujs.edu.cn (W.W.); \\ zyh11609@163.com (Y.Z.); mjk@ujs.edu.cn (M.K.O.) \\ 2 Mechanical Engineering Department, Wa Polytechnic, Wa, Upper West, Ghana \\ * Correspondence: lyj782900@ujs.edu.cn
}

Received: 8 January 2020; Accepted: 4 March 2020; Published: 7 March 2020

\begin{abstract}
This study proposed a kind of optimization design for a reversible axial-flow pump based on an ordinary one-way pump. Three-dimensional (3D) Reynolds-averaged Navier-Stokes (RANS) equations was used to predict the pump performance, and the optimized design was validated by an external characteristic test. Six main geometry parameters of an impeller and diffuser based on an orthogonal experiment were set as design variables. The efficiency and head under forward and reverse design conditions were set as the optimization objective. Based on 120 groups of sample designs obtained from Latin hypercube sampling (LHS), a two-layer artificial neural network (ANN) was used to build a non-linear function with high accuracy between the design variables and optimization objective. The optimized design was obtained from 300 groups of Pareto-optimal solutions using the non-dominated based genetic algorithm (NSGA) for multiobjective optimization. After optimization, there was a slight decrease in the forward pump efficiency and head. The reverse pump efficiency and head on the other hand was largely improved and the high efficiency range was also widened.
\end{abstract}

Keywords: reversible axial-flow pump; computational fluid dynamics; surrogate model; multiobjective optimization; two-layer ANN

\section{Introduction}

Axial-flow pump with reversible impeller can meet bidirectional pumping needs by varying the direction of the rotation of the impeller, and this has widely been used in flood control and drainage [1-3]. Due to the special blade airfoil, the flow characteristic in the reversible axial-flow pump is different from that of the conventional axial-flow pump. Thus, providing a reliable and efficient optimization design method especially for a reversible axial-flow pump is very necessary. The key point of an optimization design is accurate prediction for pump performance. In recent years, the application of computational fluid dynamics (CFD) in the research about pressure fluctuation $[4,5]$, cavitation performance [6,7] and hydraulic losses [8,9] in the different types of pump has proved numerical simulation to be an efficient method for predicting pump performance. To improve pump performance, researchers have applied the design of experiment (DOE) based on CFD for pump optimization to reduce experiment cost and save time. Long et al. [10] applied the orthogonal test to optimize the diffuser in the reactor coolant pump to improve pump performance. The optimized design 
was validated by an experiment. Liu et al. [11] used the optimal Latin hypercube sampling (LHS) method in the multicondition optimization of a mixed-flow pump and the optimization objective was chosen as weighted average efficiency at three flow rates. However, optimized design obtained from DOE is the optimal solution within the discrete design domain. Combination of the approximation model and intelligent algorithm can get the optimal solution within the continuous design domain. An approximation model is therefore being to construct a function between design variables and optimization objective. This function can then be solved by an optimization algorithm to obtain optimal optimized solutions. Pei et al. [12] therefore combined LHS, the artificial neural network (ANN) and modified particle swarm optimization (PSO) to obtain higher centrifugal pump efficiency at three flowrates. Miao et al. [13] applied the combination of neural networks and modified PSO algorithms to improve the pump efficiency and cavitation performance. Shim et al. [14,15] completed the multiobjective optimization based on approximation model and non-dominated based genetic algorithm (NSGA) to improve stability, efficiency and cavitation performance for different types of centrifugal pump. Wang et al. $[16,17]$ used different surrogate models to optimize the impeller and diffuser of a centrifugal pump based on CFD. However, there is not much optimization design on a reversible axial-flow pump.

The reversible axial blade pump generally has two-way impeller airfoils, which can be grouped into an arc, $S$-shape and polynomial curve. The $S$-shaped impeller can obtain similar pump performance under the forward and reverse condition. In some actual engineering, the pump operation time under the forward condition is much longer than that under the reverse condition. To obtain high pump efficiency under the forward condition and also to ensure that the pump meets a basic standard under the reverse condition, the bidirectional impeller airfoils are chosen as an asymmetric arc or polynomial curve. Ma $[18,19]$ investigated the pressure fluctuation in a reversible axial-flow pump with an S-shaped impeller, and compared the inner flow in an S-shaped and arc bidirectional impeller. It provided some useful suggestions for hydraulic design of a reversible axial-flow pump. Jung [20] combined an orthogonal experimental design, response surface model (RSM) and feasible direction method to optimize the arc two-way impeller with a symmetric chord airfoil.

In this study, the one-way axial-flow pump was selected as the basis of combination optimization design for a two-way impeller and diffuser. The optimization objective was to improve the reverse pump performance and maintain forward pump performance at a certain level. The 3D Reynolds-averaged Navier-Stokes (RANS) equation was solved to predict pump performance and six geometry parameters of the impeller and diffuser were set as the design variables based on an orthogonal experimental design. The relationship between design variables and optimization objective was constructed with 120 groups of sample points using LHS and two-layer ANN. Finally, the optimized design was obtained based on 300 groups of Pareto-optimal solutions by using NSGA, and the external characteristic experiment of optimized design was performed to verify the optimization results.

\section{Numerical Method}

\subsection{Computational Model and Mesh}

The original design is a one-way pump, which consists of a straight pipe, a bidirectional impeller, a diffuser and an elbow as shown in Figure 1. The optimized pump is a reversible axial-flow pump consisting of a straight pipe, a two-way impeller, a diffuser and an elbow. The main design parameters of the original design are listed in Table 1, and the specific speed can be calculated by Equation (1).

$$
n_{s}=\frac{3.65 N Q^{0.5}}{H^{0.75}}
$$

where $n_{S}$ is the specific speed, $N$ is rotational speed, $Q$ is the volume flow rate and $H$ is the head. 


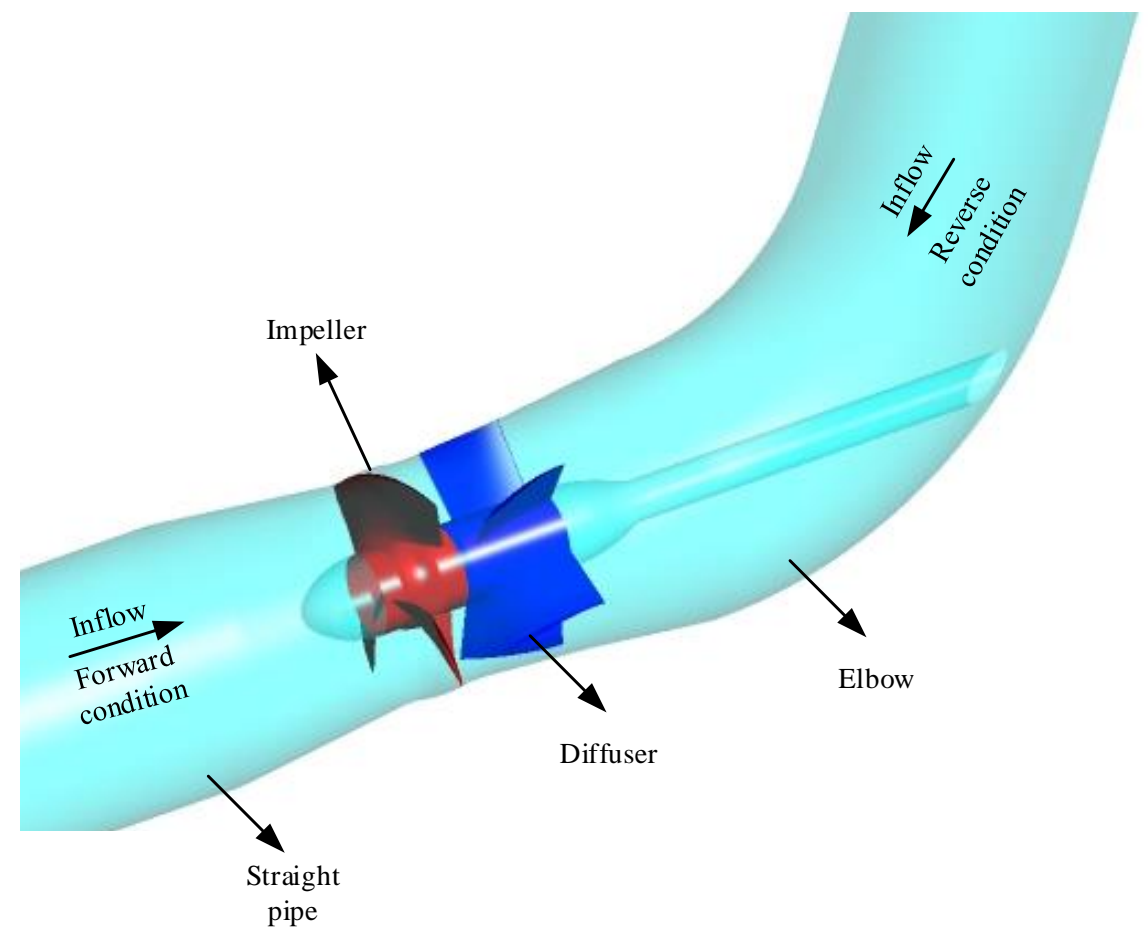

Figure 1. 3D model of the original design.

Table 1. Main parameters of the original design.

\begin{tabular}{cccc}
\hline & \multicolumn{2}{c}{ Design Parameters } \\
\hline Rated flow rate $\left(\mathrm{m}^{3} / \mathrm{s}\right)$ & 0.4 & Rotational speed $(\mathrm{r} / \mathrm{min})$ & 1450 \\
Head on rated flow rate $(\mathrm{m})$ & 3.87 & Specific speed & 1213.1 \\
\hline \multicolumn{2}{c}{ Geometry Parameters } \\
\hline Impeller blade number & 3 & Diffuser blade number & 5 \\
Impeller diameter $(\mathrm{mm})$ & 300 & Outlet diameter of diffuser $(\mathrm{mm})$ & 312.8 \\
\hline & \multicolumn{2}{c}{ Optimization Parameters } & 78.0 \\
\hline Forward design flow rate $\left(\mathrm{m}^{3} / \mathrm{s}\right)$ & 0.36 & Efficiency on forward design flow rate $(\%)$ & 51.91 \\
\hline Reverse design flow rate $\left(\mathrm{m}^{3} / \mathrm{s}\right)$ & 0.3 & Efficiency on reverse design flow rate $(\%)$ & \\
\hline
\end{tabular}

The straight pipe and elbow were set as an inflow pipe and outflow pipe respectively, under the forward operation. In the reverse operation, the straight pipe and elbow were set as the outlet pipe and inlet pipe respectively. Figure 2 shows the mesh of the entire computational domain. The hexahedral mesh of the impeller and diffuser was generated by TurboGrid. The hexahedral mesh of the other components was obtained by preprocessing software of computer aided engineering (CAE), ICEM CFD, which is a module in the commercial software ANASYS. This software can generate a hexahedral mesh semi-automatically and generate a tetrahedral mesh automatically. Figure 3 shows the grid independence analysis of the original design for the impeller and diffuser. In this figure, $\eta_{1}$ stands for pump efficiency under a forward design flow rate, $\eta_{2}$ stands for pump efficiency under a reverse design flow rate. Figure 3a,b shows the tendency of $\eta_{1}$ and $\eta_{2}$ with a grid number of the impeller increasing. The maximum relative deviation was smaller than $0.08 \%$ when the grid number was greater than $1.25 \times 10^{6}$. Figure $3 c$,d shows the tendency of $\eta_{1}$ and $\eta_{2}$ with a grid number of the diffuser increasing. The maximum relative deviation was smaller than $0.16 \%$ when the grid number was greater than $1.47 \times 10^{6}$. Hence, the number of nodes of the inlet section, impeller, diffuser and outlet section were finally chosen as approximately $0.74 \times 10^{6}, 1.25 \times 10^{6}, 1.47 \times 10^{6}$ and $0.95 \times 10^{6}$ respectively. $\mathrm{Y}+$ is a dimensionless number and it can define the distance from the wall to the first node. The average $\mathrm{Y}+$ 
of any component can be obtained by calculating the mean $Y+$ values from each node on all walls. The average $\mathrm{Y}+$ of the straight pipe without the inlet section, impeller, diffuser and elbow pipe without the outlet section were $21.8,24.3,13.52$ and 45.4 respectively.

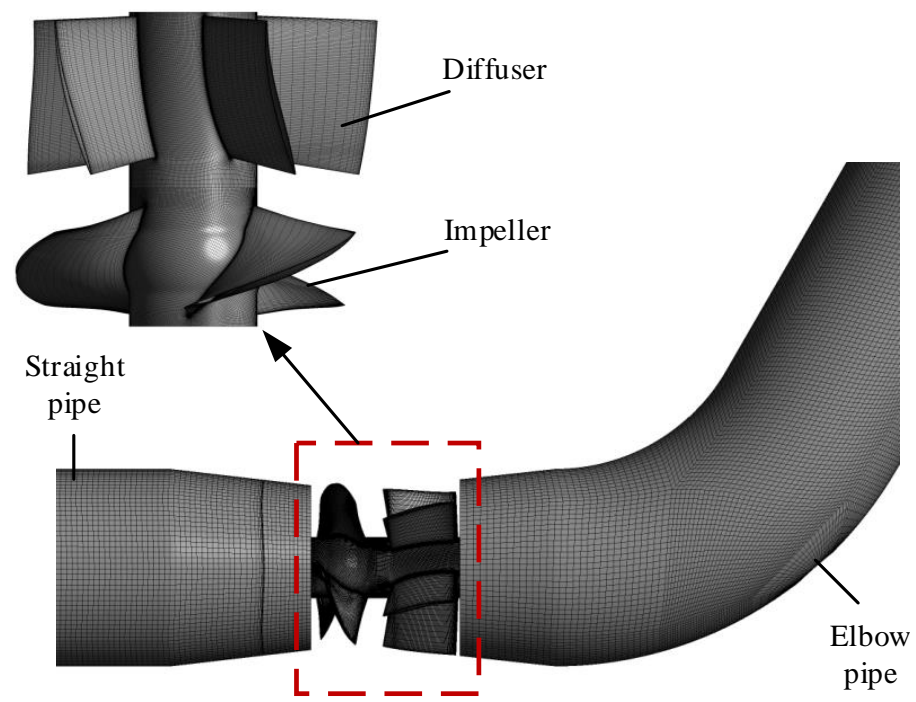

Figure 2. Mesh of the original design.

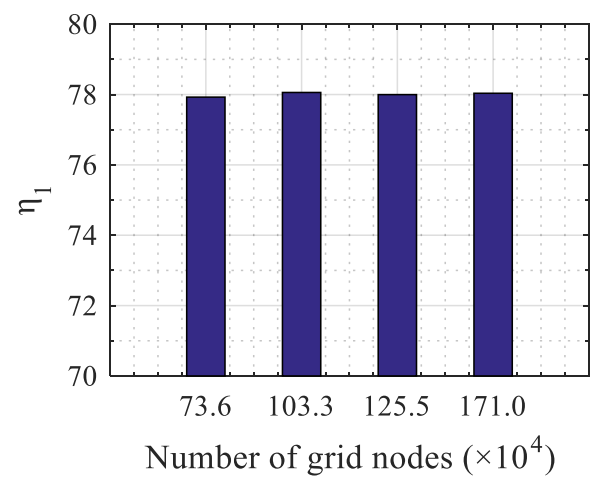

(a)

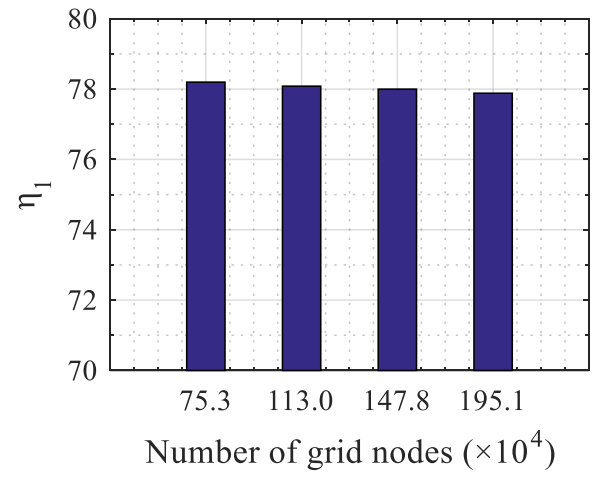

(c)

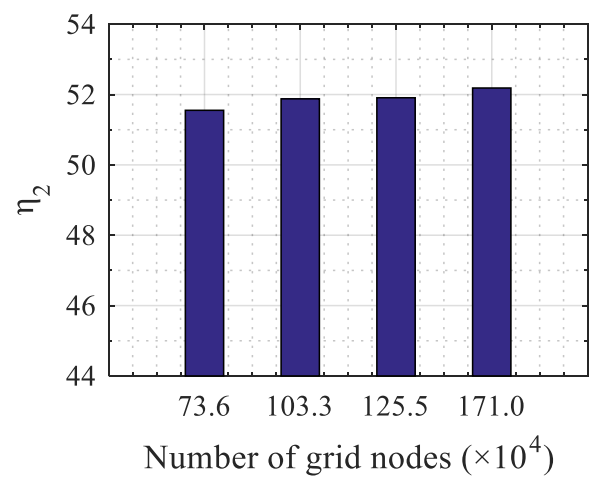

(b)

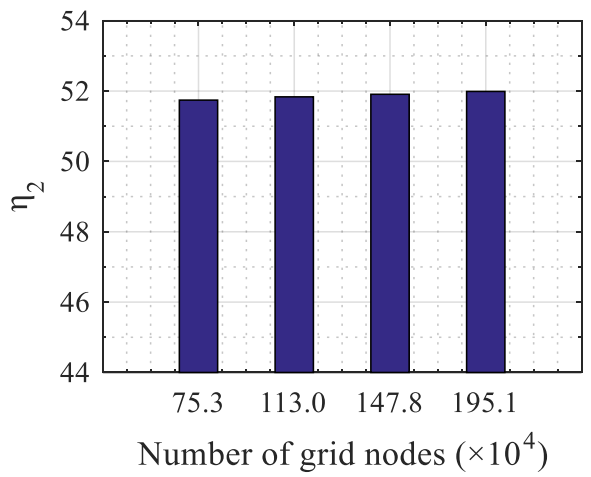

(d)

Figure 3. Grid independence of the original design for the (a) impeller under a forward design flowrate, (b) impeller under a reverse design flow rate, (c) diffuser under a forward design flowrate and (d) diffuser under a reverse design flow rate.

\subsection{Numerical Setup}

The $k-\omega$ formulation has good performance near the wall treatment for low Reynolds number computations, so it was applied widely in the numerical simulation [21] in the pump. In addition, 
the $k-\omega$ based Shear-Stress-Transport (SST) model with an automatic wall treatment can present a smooth transition from a low-Reynolds number form to a wall function formulation, which shows less sensitivity to $\mathrm{Y}+$ [22]. So, it was used to solve a steady 3D RANS equation in this study. The governing equation was defined as:

$$
\begin{gathered}
\frac{\partial \bar{v}_{j}}{\partial x_{i}}=0 \\
\frac{\partial\left(\rho \bar{v}_{i}\right)}{\partial t}+\frac{\partial\left(\rho \bar{v}_{i} \bar{v}_{j}\right)}{\partial x_{j}}=-\frac{\partial \bar{p}}{\partial x_{i}}+\frac{\partial}{\partial x_{j}}\left(\mu \frac{\partial \bar{v}_{i}}{\partial x_{j}}-\overline{\rho v^{\prime}{ }_{i} v_{j}^{\prime}}\right)+\rho f_{i}
\end{gathered}
$$

where $\bar{v}$ is the time-average speed and $\bar{p}$ is the time-average pressure. $\rho$ is the fluid density. $\mu$ stands for the dynamic viscosity and $\overline{\rho v^{\prime}{ }_{i} v^{\prime}}{ }_{j}$ represents the Reynolds stress. $\rho f_{i}$ is the source item.

The reference pressure is $1 \mathrm{~atm}$. The inlet boundary condition was set as the 'Mass Flow Rate' under a forward operation. The outlet boundary condition was set as 'Static Pressure' and the outlet static pressure was initially specified as 0 . In the reverse operation, the inlet boundary condition was also set as the 'Mass Flow Rate'. However, the outlet boundary condition was set as the 'Opening' due to flow recirculation and the opening pressure was initially specified as 0 . The interface condition between the impeller and stator was set as a 'Frozen rotor' (the relative position of the impeller and stator across the interface was fixed through calculation). The interface condition between stators was set as 'None'. The wall boundary condition was considered as smooth with no slip. The convergence criteria and advection scheme were set as $1 \times 10^{4}$ and 1st-order upwind respectively. The numerical calculation was to be stopped when it achieved convergence criteria or periodic stability (few calculated results shown periodic oscillations, but the amplitude of efficiency is less than $0.5 \%$.).

\section{Optimization Procedure}

The optimization procedure for the improvement of pump efficiency and the head is shown in Figure 4. Firstly, the shape of the impeller blade and diffuser blade was controlled by the airfoil section. The main geometric parameters of the airfoil section were set as design variables and described in detail in Section 3.2. In order to reduce the design variables of the diffuser to improve the optimization speed, the orthogonal experimental design was applied to obtain the effect of main geometric parameters on the optimization objective. Thirdly, LHS was used to construct 120 groups of sample designs in design space. The CFD results of the optimization objective were obtained by CFX automatically executed by MATLAB as shown in Figure 5. A two-layer ANN was designed to construct the non-linear function between the design variables and optimization objective. Finally, the 300 groups of Pareto-optimal solutions were obtained by NSGA. Optimized design was based on one of these solutions and verified by experiment data.

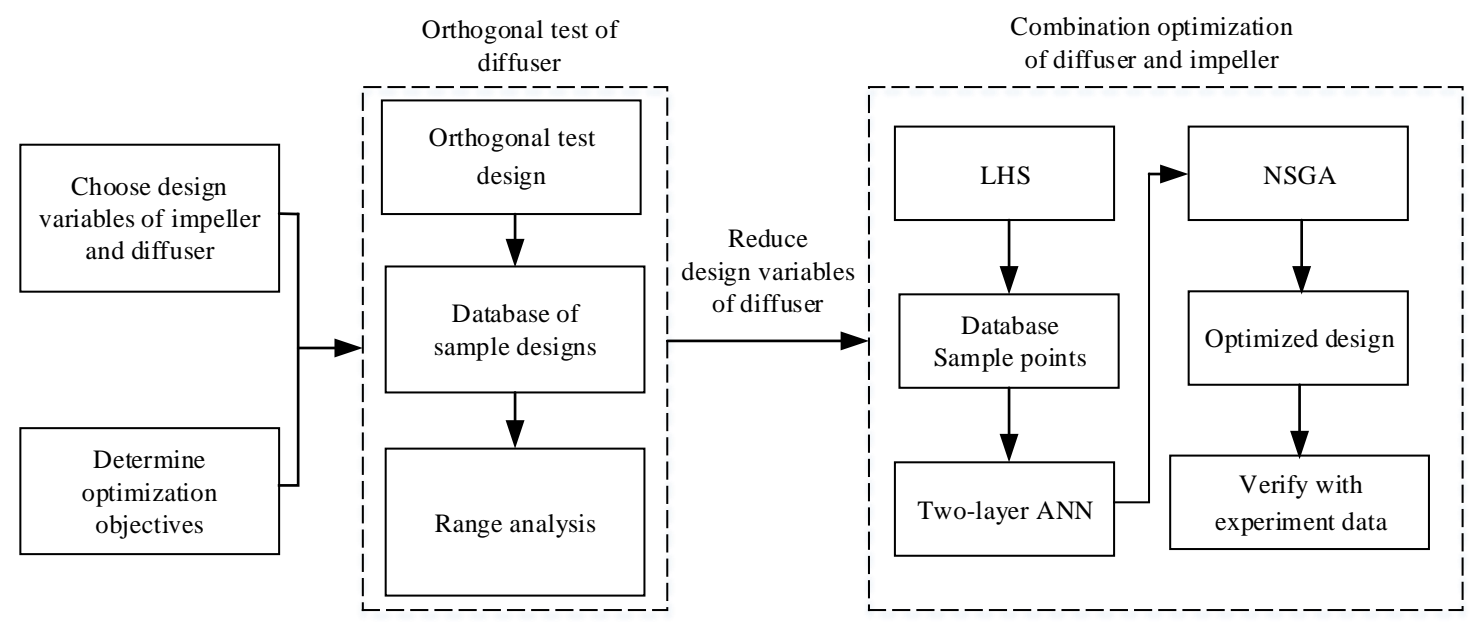

Figure 4. The optimization procedure of the reversible axial-flow pump. 


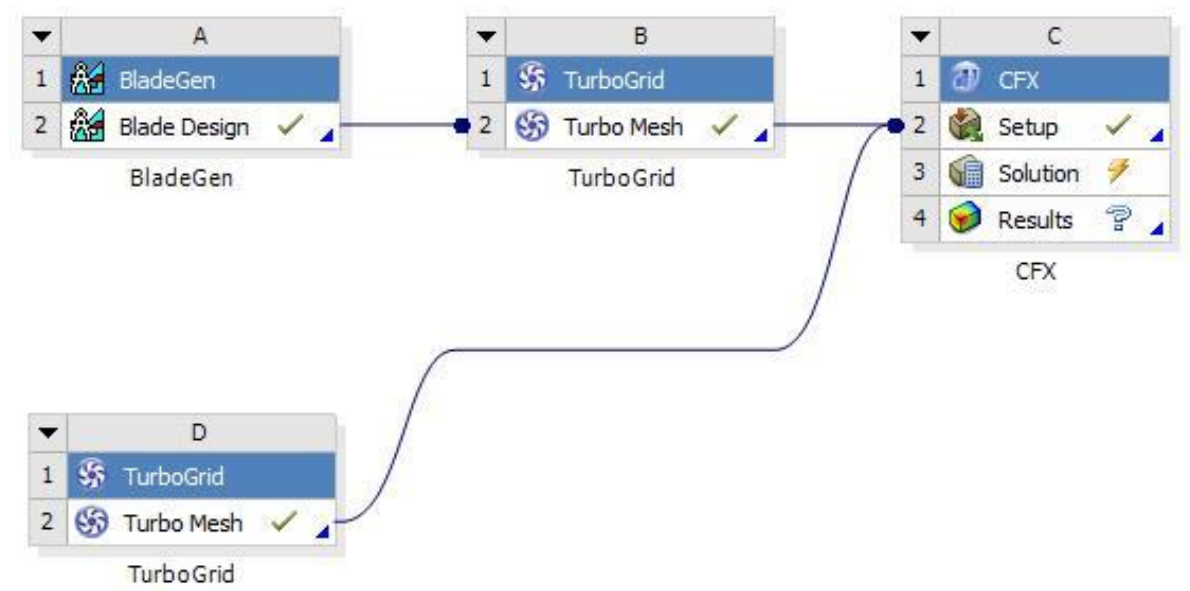

Figure 5. Optimization flow chart in the ANSYS Workbench.

\subsection{Optimization Functions}

According to engineering requirements, the forward design flow rate was chosen as $0.36 \mathrm{~m}^{3} / \mathrm{s}$ and the reverse design flow rate was chosen as $0.3 \mathrm{~m}^{3} / \mathrm{s}$. The optimization objectives can be described as follow:

$$
\text { Minimum : } \mathbf{Z}(\boldsymbol{x})=\left\{\mathbf{Z}_{1}(x), \mathbf{Z}_{2}(x), \mathbf{Z}_{3}(x)\right\}
$$

where $x$ is a vector of design variables. $Z$ stands for a vector of three optimization objectives $Z_{1}, Z_{2}$ and $Z_{3}$.

In this study, three optimization objectives need be minimized to improve hydraulic performance to meet the engineering requirement. The first optimization objective $Z_{1}$ is the negative hydraulic efficiency under the forward design flow rate. The second optimization objective $Z_{2}$ is the negative hydraulic efficiency under the reverse design flow rate. The third optimization objective $Z_{3}$ is the negative sum of the head under a forward design flow rate and head under a reverse design flow rate. Three objective functions were defined as:

$$
\begin{gathered}
Z_{1}=-\eta_{1} \\
Z_{2}=-\eta_{2} \\
Z_{3}=-H_{3}=-\left(H_{1}+H_{2}\right)
\end{gathered}
$$

where $\eta_{1}$ and $H_{1}$ are hydraulic efficiency and a head under the forward design flow rate. $\eta_{2}$ and $H_{2}$ are hydraulic efficiency and the head under the reverse design flow rate. $H_{3}$ is the sum of $H_{1}$ and $H_{2}$.

The hydraulic efficiency $\eta$ and head $H$ can be calculated by the equations as follow:

$$
\begin{gathered}
\eta=\frac{\left(P_{\text {out }}-P_{\text {in }}\right) Q}{T N} \\
H=\frac{P_{\text {out }}-P_{\text {in }}}{\rho g}
\end{gathered}
$$

where $P_{\text {out }}$ is the total pressure in the outlet section, $P_{\text {in }}$ is the total pressure in the inlet section. $Q$ is the volume flow rate. $T$ is the torque on the pump shaft during pump operation. $N$ is the forward rotation speed of the pump shaft. $\rho$ is the fluid density and $g$ is the gravitational acceleration.

\subsection{Geometry Variables}

Figure 6a shows the impeller shape was defined by five airfoil sections (I-I, II-II, III-III, IV-IV and $V-V$ ) obtained by radial equidistant division. The main geometry parameters of every airfoil section are the length of the chord line $L$, blade angle (angle between the chord line and horizontal line) $\theta$, deformation of the mean line (midpoint connection of the local geometric thickness of the airfoil section 
along chord line) and thickness distribution along the chord line, as shown in Figure 6b. In this study, every airfoil section was designed based on the NACA 65 series. So, the deformation of the mean line in every section could calculate the form lift coefficient $\sigma$, which was obtained from the camber angle $\Phi$. The definition of $\Phi$ was shown in Figure $6 c$ and the deformation function of the mean line was described in detail as follows [23]:

$$
\begin{gathered}
\sigma=\frac{2 \pi}{\ln (2)} \tan \frac{\Phi}{4} \\
\Phi=\lambda-\varepsilon \\
\frac{y_{c}}{L}=-\frac{\sigma}{4 \pi}\left[\left(1-\frac{x_{c}}{L}\right) \ln \left(1-\frac{x_{c}}{L}\right)+\frac{x_{c}}{L_{i}} \ln \left(\frac{x_{c}}{L}\right)\right]
\end{gathered}
$$

where $\sigma$ is the theoretical lift coefficient of the airfoil, $\Phi$ is the camber angle. $\lambda$ and $\varepsilon$ are the inlet angle and outlet angle of the skeleton line. $y_{c}$ is the ordinate of the airfoil profile (direction perpendicular to chord line). $x_{c}$ is the abscissa of the airfoil profile (direction parallel to the chord line).

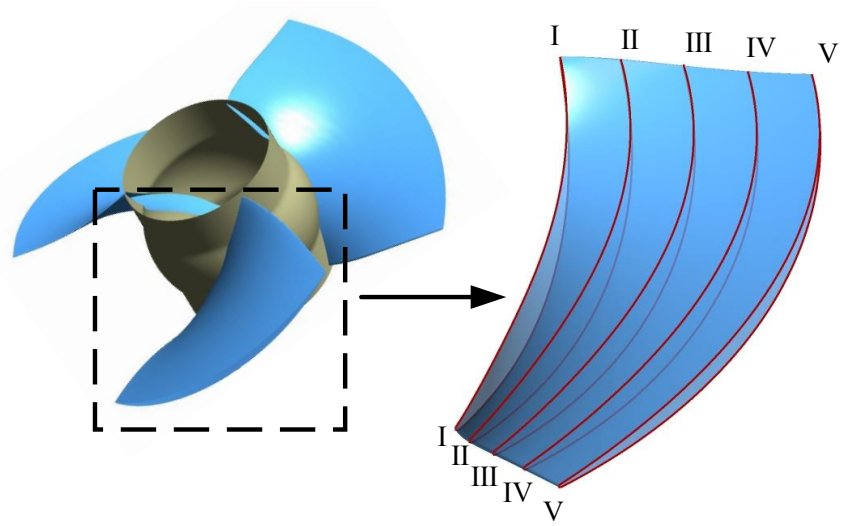

(a)

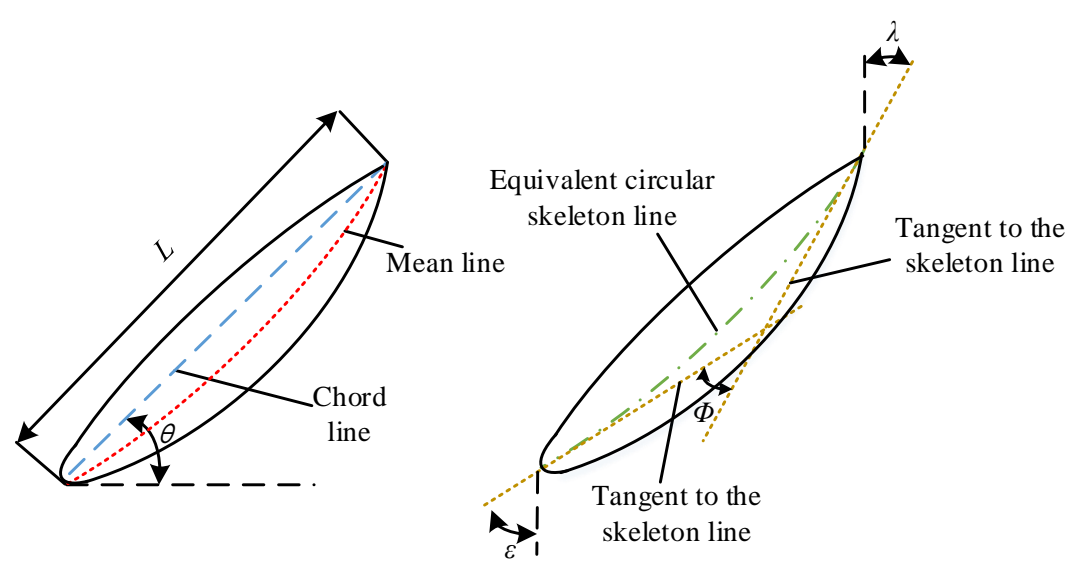

(b)

(c)

Figure 6. The definition of the main geometry variables of the impeller: (a) definition of the airfoil section, (b) main geometry variables of the airfoil and (c) the rest of the geometry variables.

In this hydraulic design, the thickness distribution and blade angle $\theta$ were predetermined, so the shape of the airfoil section was controlled by the length of the chord line $L$ and camber $\Phi$. In order to improve the optimization speed, the design variables of the impeller was only chosen from the chord length and camber angle in section $I-I$ and section $V-V: L_{1}, L_{5}, \Phi_{1}$ and $\Phi_{5}$. The $L$ and $\Phi$ in the rest sections were determined by the equation as follow:

$$
\begin{gathered}
L_{\kappa}=\frac{\left(L_{5}-L_{1}\right)}{4} \times \kappa+L_{1} \\
\Phi_{\kappa}=\frac{\left(\Phi_{5}-\Phi_{1}\right)}{4} \times \kappa+\Phi_{1}
\end{gathered}
$$


where, $\kappa$ is the section number of the impeller. $\Phi_{1}$ and $\Phi_{5}$ are the camber angle in section $I-I$ and section $V-V . L_{1}$ and $L_{5}$ are the length of the chord line in section $I-I$ and section $V-V$.

Figure 7 a shows the diffuser shape was defined by five airfoil sections (I-I, II-II, III-III, IV-IV and $V-V)$ obtained by radial equidistant division. Figure $7 \mathrm{~b}$ shows that every airfoil section was defined by the inlet angle $\alpha$, outlet angle $\beta$, blade height $\zeta$ and wrap angle $\varphi$. In this study, the outlet angle $\beta$ and blade height $\zeta$ were predetermined based on previous engineering research. Only the wrap angle $\varphi$ and inlet angle $\alpha$ in section $I-I$ and section $V$ - $V$ were set as geometry variables. The $\varphi$ and $\alpha$ in the rest of the sections were calculated as follow:

$$
\begin{aligned}
& \varphi_{\varsigma}=\frac{\left(\varphi_{5}-\varphi_{1}\right)}{4} \times \varsigma+\varphi_{1} \\
& \alpha_{\varsigma}=\frac{\left(\alpha_{5}-\alpha_{1}\right)}{4} \times \varsigma+\alpha_{1}
\end{aligned}
$$

where, $\varsigma$ is the section number of the diffuser. $\varphi_{5}$ is the wrap angle in section $V-V$ and $\varphi_{1}$ is the wrap angle in section I-I. $\alpha_{5}$ is the inlet angle in section $V-V$ and $\alpha_{1}$ is the inlet angle in section I-I.

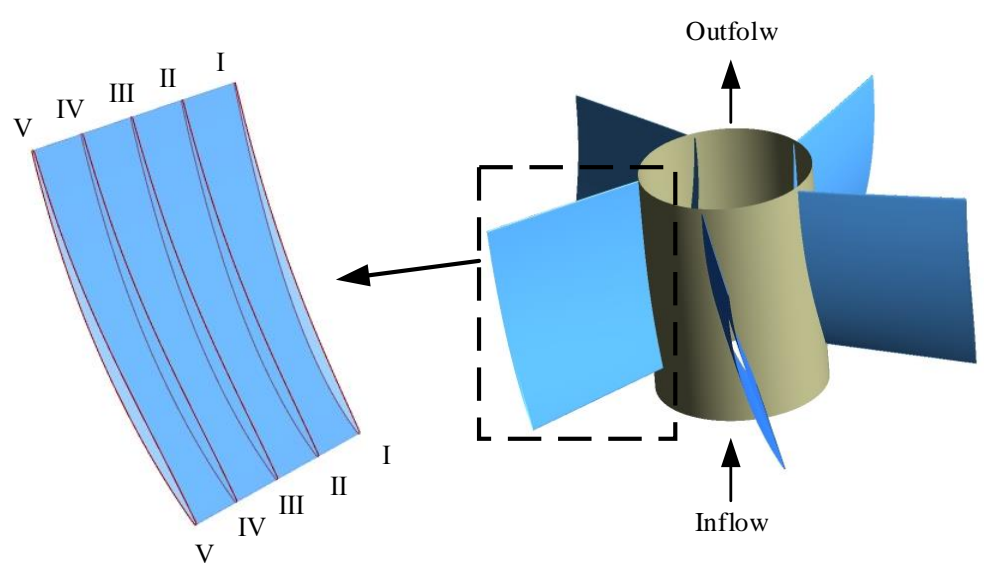

(a)
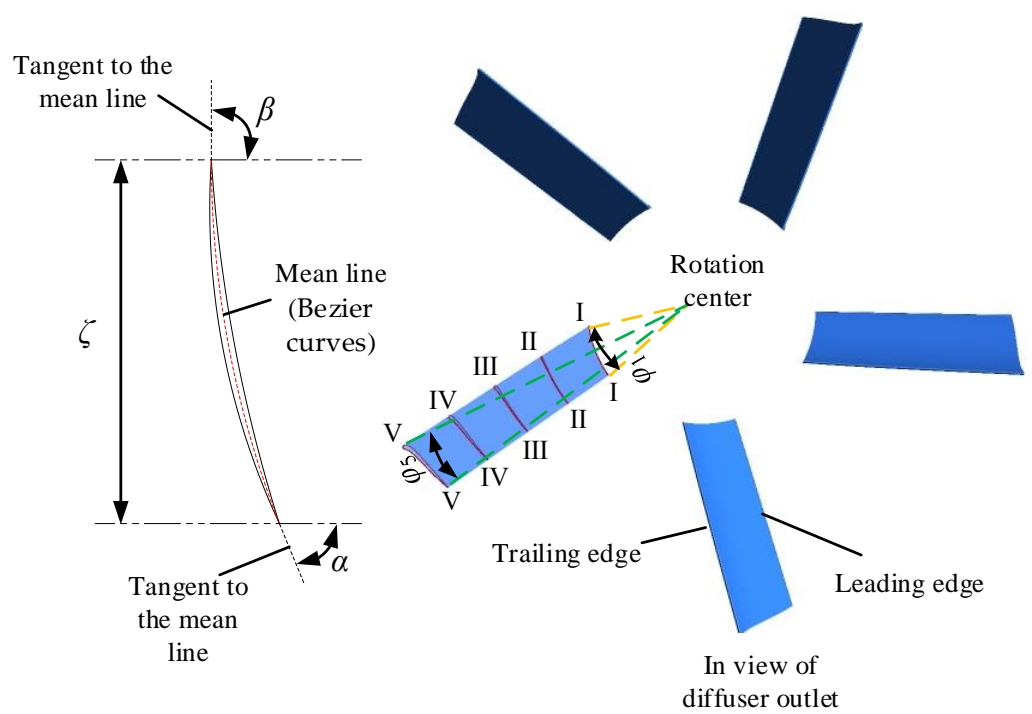

(b)

Figure 7. The definition of the main geometry variables of the diffuser: (a) definition of the airfoil section and (b) main geometry variables of the airfoil.

\subsection{Orthogonal Test Design of Diffuser}

According to Section 3.1, the impeller shape was controlled by $L_{1}, L_{5}, \Phi_{1}$ and $\Phi_{5}$, and the diffuser shape was controlled by $\varphi_{1}, \varphi_{5}, \alpha_{1}$ and $\alpha_{5}$. The focus was to optimize the impeller and diffuser 
at the same time in this study. However, if all these eight geometry variables were set as design variables, the computing resource consumption would increase and optimization speed would decline. So, the number of design variables had to be reduced. Considering the importance of impellers for hydraulic performance, the total number of design variables should be reduced by reducing geometry variables of the diffuser.

Orthogonal test design [24] is a kind of economical and fast design method, which was used for a multi factor with a different level. Some representative designs with evenly dispersed and comparable were selected. Then the range analysis based on these representative designs was obtained to evaluate the qualitative contribution of each geometry variables on the optimization objective. Finally, the geometry variables that were influential on the optimization objective were selected as design variables.

\subsubsection{Orthogonal Test Scheme}

Table 2 shows that $\varphi_{1}, \varphi_{5}, \alpha_{1}$ and $\alpha_{5}$ were set as factor A, B, C and D respectively. Each of these factors was considered at five levels in the orthogonal test. Table 3 shows the orthogonal test scheme, and the correspondence between the serial number of the factor and geometric parameters. Three optimization objectives under the orthogonal test scheme were obtained by numerical simulation.

Table 2. Level of the orthogonal factor.

\begin{tabular}{ccccc}
\hline & \multicolumn{4}{c}{ Factor } \\
\cline { 2 - 5 } Level & A & B & C & D \\
& $\alpha_{5}$ & $\alpha_{1}$ & $\varphi_{5}$ & $\varphi_{1}$ \\
\hline Level 1 & 70 & 85 & 26 & 4.5 \\
Level 2 & 65 & 80 & 30 & 6.5 \\
Level 3 & 60 & 75 & 34 & 8.5 \\
Level 4 & 55 & 70 & 38 & 10.5 \\
Level 5 & 50 & 65 & 42 & 12.5 \\
\hline
\end{tabular}

Table 3. Orthogonal test scheme.

\begin{tabular}{|c|c|c|c|c|c|c|c|c|c|c|c|}
\hline \multirow{2}{*}{$\begin{array}{c}\text { Test } \\
\text { Number }\end{array}$} & \multicolumn{4}{|c|}{ Factor } & \multicolumn{4}{|c|}{ Corresponding Parameter } & \multicolumn{3}{|c|}{ Optimization Objective } \\
\hline & $A$ & B & C & $D$ & $\alpha_{5}$ & $\alpha_{1}$ & $\varphi_{5}$ & $\varphi_{1}$ & $\eta_{1} /(\%)$ & $\eta_{2} /(\%)$ & $H_{3} /(\mathrm{m})$ \\
\hline 1 & $A_{1}$ & $B_{1}$ & $C_{1}$ & $D_{1}$ & 70 & 85 & 26 & 4.5 & 73.62 & 54.04 & 7.30 \\
\hline 2 & $A_{1}$ & $B_{2}$ & $C_{2}$ & $D_{2}$ & 70 & 80 & 30 & 6.5 & 75.43 & 53.30 & 7.21 \\
\hline 3 & $A_{1}$ & $B_{3}$ & $C_{3}$ & $D_{3}$ & 70 & 75 & 34 & 8.5 & 76.86 & 52.30 & 7.12 \\
\hline 4 & $A_{1}$ & $B_{4}$ & $C_{4}$ & $D_{4}$ & 70 & 70 & 38 & 10.5 & 77.85 & 51.12 & 6.99 \\
\hline 5 & $A_{1}$ & $B_{5}$ & $C_{5}$ & $D_{5}$ & 70 & 65 & 42 & 12.5 & 78.90 & 49.69 & 6.88 \\
\hline 6 & $A_{2}$ & $B_{1}$ & $\mathrm{C}_{2}$ & $D_{3}$ & 65 & 85 & 30 & 8.5 & 75.98 & 52.52 & 7.17 \\
\hline 7 & $A_{2}$ & $B_{2}$ & $C_{3}$ & $D_{4}$ & 65 & 80 & 34 & 10.5 & 77.37 & 51.52 & 7.06 \\
\hline 8 & $A_{2}$ & $B_{3}$ & $C_{4}$ & $D_{5}$ & 65 & 75 & 38 & 12.5 & 78.79 & 50.31 & 6.98 \\
\hline 9 & $A_{2}$ & $B_{4}$ & $C_{5}$ & $D_{1}$ & 65 & 70 & 42 & 4.5 & 77.75 & 51.23 & 6.99 \\
\hline 10 & $A_{2}$ & $B_{5}$ & $C_{1}$ & $D_{2}$ & 65 & 65 & 26 & 6.5 & 77.32 & 51.96 & 7.03 \\
\hline 11 & $A_{3}$ & $B_{1}$ & $C_{3}$ & $D_{5}$ & 60 & 85 & 34 & 12.5 & 77.74 & 50.73 & 7.00 \\
\hline 12 & $A_{3}$ & $B_{2}$ & $C_{4}$ & $D_{1}$ & 60 & 80 & 38 & 4.5 & 76.43 & 51.75 & 7.03 \\
\hline 13 & $A_{3}$ & $B_{3}$ & $C_{5}$ & $D_{2}$ & 60 & 75 & 42 & 6.5 & 78.22 & 50.53 & 6.94 \\
\hline 14 & $A_{3}$ & $B_{4}$ & $C_{1}$ & $D_{3}$ & 60 & 70 & 26 & 8.5 & 77.92 & 51.34 & 7.01 \\
\hline 15 & $A_{3}$ & $B_{5}$ & $C_{2}$ & $D_{4}$ & 60 & 65 & 30 & 10.5 & 79.38 & 49.85 & 6.92 \\
\hline 16 & $A_{4}$ & $B_{1}$ & $C_{4}$ & $D_{2}$ & 55 & 85 & 38 & 6.5 & 76.96 & 50.95 & 6.98 \\
\hline 17 & $A_{4}$ & $B_{2}$ & $C_{5}$ & $D_{3}$ & 55 & 80 & 42 & 8.5 & 78.45 & 49.66 & 6.87 \\
\hline 18 & $A_{4}$ & $B_{3}$ & $C_{1}$ & $D_{4}$ & 55 & 75 & 26 & 10.5 & 78.54 & 50.45 & 6.96 \\
\hline 19 & $A_{4}$ & $B_{4}$ & $C_{2}$ & $D_{5}$ & 55 & 70 & 30 & 12.5 & 79.82 & 48.90 & 6.85 \\
\hline 20 & $A_{4}$ & $B_{5}$ & $C_{3}$ & $D_{1}$ & 55 & 65 & 34 & 4.5 & 77.64 & 49.81 & 6.79 \\
\hline 21 & $A_{5}$ & $B_{1}$ & $C_{5}$ & $D_{4}$ & 50 & 85 & 42 & 10.5 & 78.77 & 48.56 & 6.80 \\
\hline 22 & $A_{5}$ & $B_{2}$ & $C_{1}$ & $D_{5}$ & 50 & 80 & 26 & 12.5 & 79.06 & 49.67 & 6.91 \\
\hline 23 & $A_{5}$ & $B_{3}$ & $C_{2}$ & $D_{1}$ & 50 & 75 & 30 & 4.5 & 77.19 & 50.54 & 6.87 \\
\hline 24 & $A_{5}$ & $B_{4}$ & $C_{3}$ & $D_{2}$ & 50 & 70 & 34 & 6.5 & 78.47 & 49.07 & 6.76 \\
\hline 25 & $A_{5}$ & $B_{5}$ & $C_{4}$ & $D_{3}$ & 50 & 65 & 38 & 8.5 & 80.34 & 47.07 & 6.69 \\
\hline
\end{tabular}




\subsubsection{Range Analysis}

$K_{i}, \bar{K}_{i}$ and $R$ were set as the evaluation index of the range analysis. $K_{i}$ is the sum value of each level for a certain factor. $\bar{K}_{i}$ is the average value of $K_{i}$, which can reflect the optimal levels of a certain factor for $\eta_{1}, \eta_{2}$ and $H_{3}$.

$R$ stands for the maximum range that the optimization objective changes as the factor changes. The larger the range size, the greater the qualitative contribution of the factor to the optimization objective. These evaluation indexes can be calculated as follows:

$$
\begin{gathered}
K_{i}=\sum_{j=1}^{5} Y_{i j} \\
\bar{K}_{i}=\frac{1}{5} \sum_{j=1}^{5} Y_{i j} \\
R=\max \left(\bar{K}_{1}, \bar{K}_{2}, \bar{K}_{3}, \bar{K}_{4}, \bar{K}_{5}\right)-\min \left(\bar{K}_{1}, \bar{K}_{2}, \bar{K}_{3}, \bar{K}_{4}, \bar{K}_{5}\right)
\end{gathered}
$$

where $Y_{i j}$ stands for the optimization objective of the $i$ factor in level $j . K_{i}$ is the sum value of each level for a certain factor. $\bar{K}_{i}$ is the average value of $K_{i}$.

Table 4 shows the range analysis of the pump efficiency under a forward design condition. The qualitative contribution of the factor to $\eta_{1}$ according to $R$ was arranged from high to low: $D, A, B$ and $C$. Table 5 shows the range analysis of the pump efficiency under the reverse design condition. The qualitative contribution of the factor to $\eta_{2}$ according to $R$ was arranged from high to low: A, B, D and $C$. Table 6 shows the range analysis of the sum of the head under the forward design condition and the head under the reverse design condition. The $R$ was so little from the range analysis of $H_{3}$. So, the qualitative contribution of the factor to $H_{3}$ was not obvious. In summary, factor $\mathrm{D}\left(\varphi_{1}\right)$ and $\mathrm{A}\left(\alpha_{5}\right)$ had the most important influence on $\eta_{1}$ and $\eta_{2}$ based on $R$. So, $\varphi_{1}$ and $\alpha_{5}$ were set as design variables in the next combination optimization of the impeller and diffuser.

Table 4. Range analysis of $\eta_{1}$

\begin{tabular}{ccccc}
\hline Test Indices & D & A & B & C \\
\hline$K_{1}$ & 382.63 & 382.66 & 383.08 & 386.45 \\
$K_{2}$ & 386.41 & 387.21 & 386.73 & 387.81 \\
$K_{3}$ & 389.54 & 389.70 & 389.60 & 388.08 \\
$K_{4}$ & 391.90 & 391.41 & 391.81 & 390.37 \\
$K_{5}$ & 394.32 & 393.83 & 393.59 & 392.10 \\
$\bar{K}_{1}$ & 76.53 & 76.53 & 76.62 & 77.29 \\
$\bar{K}_{2}$ & 77.28 & 77.44 & 77.35 & 77.56 \\
$\bar{K}_{3}$ & 77.91 & 77.94 & 77.92 & 77.62 \\
$\bar{K}_{4}$ & 78.38 & 78.28 & 78.36 & 78.07 \\
$\bar{K}_{5}$ & 78.86 & 78.77 & 78.72 & 78.42 \\
$R$ & 2.34 & 2.23 & 2.10 & 1.13 \\
\hline
\end{tabular}

Table 5. Range analysis of $\eta_{2}$

\begin{tabular}{ccccc}
\hline Test Indices & $\mathbf{A}$ & $\mathbf{B}$ & $\mathbf{D}$ & $\mathbf{C}$ \\
\hline$K_{1}$ & 260.45 & 256.80 & 257.38 & 257.45 \\
$K_{2}$ & 257.55 & 255.90 & 255.81 & 255.10 \\
$K_{3}$ & 254.20 & 254.14 & 252.88 & 253.44 \\
$K_{4}$ & 249.76 & 251.65 & 251.50 & 251.21 \\
$K_{5}$ & 244.91 & 248.38 & 249.30 & 249.68 \\
$\bar{K}_{1}$ & 52.09 & 51.36 & 51.48 & 51.49 \\
$\bar{K}_{2}$ & 51.51 & 51.18 & 51.16 & 51.02 \\
$\bar{K}_{3}$ & 50.84 & 50.83 & 50.58 & 50.69 \\
$\bar{K}_{4}$ & 49.95 & 50.33 & 50.30 & 50.24 \\
$\bar{K}_{5}$ & 48.98 & 49.68 & 49.86 & 49.94 \\
$R$ & 3.11 & 1.68 & 1.62 & 1.55 \\
\hline
\end{tabular}


Table 6. Range analysis of $H_{3}$

\begin{tabular}{ccccc}
\hline Test Indices & $\mathbf{A}$ & $\mathbf{B}$ & $\mathbf{C}$ & $\mathbf{D}$ \\
\hline$K_{1}$ & 35.51 & 35.25 & 35.22 & 34.98 \\
$K_{2}$ & 35.24 & 35.09 & 35.03 & 34.93 \\
$K_{3}$ & 34.90 & 34.87 & 34.72 & 34.85 \\
$K_{4}$ & 34.45 & 34.60 & 34.67 & 34.73 \\
$K_{5}$ & 34.03 & 34.31 & 34.49 & 34.62 \\
$\bar{K}_{1}$ & 7.10 & 7.05 & 7.04 & 7.00 \\
$\bar{K}_{2}$ & 7.05 & 7.02 & 7.01 & 6.99 \\
$\bar{K}_{3}$ & 6.98 & 6.97 & 6.94 & 6.97 \\
$\bar{K}_{4}$ & 6.89 & 6.92 & 6.93 & 6.95 \\
$\bar{K}_{5}$ & 6.81 & 6.86 & 6.90 & 6.92 \\
$R$ & 0.30 & 0.19 & 0.14 & 0.07 \\
\hline
\end{tabular}

\subsection{Latin Hypercube Sampling}

LHS [25] is a method of approximately random sampling. This method can divide the sampling unit into different layers and randomly sample from different parts based on a certain ratio. In this study LHS was applied to obtain design points to construct the surrogate model. A lack of enough design points can result in losing some regions of space design since large sample properties of simulations are required during LHS. So, the LHS was used to generate 120 groups of design points to ensure a full display of the design space. Among these sample points, 90 groups of design points were selected as training data and 30 groups of design points were used as test data.

\subsection{Multilayer Artificial Neural Networks}

The approximation model can build the function between the design variables and optimization objective based on the design points obtained from DOE. The optimization objective can be predicted by this function. Common approximation models include RSM [26], Kriging Model [27], radial basis function (RBF) [28] and ANN [29].

ANN is a kind of computing system inspired by the biological neural networks. It can be divided into several layers and the function between inputs and outputs can be constructed through layer-by-layer mapping. As shown in Figure 8, the first layer is called the input layer and the last layer is called the output layer. Layers between the input layer and output layer are collectively called the hidden layers. The mapping relationship between layers is called the transfer function. The multilayer ANN with more than one hidden layer can build a strong non-linear function between inputs and outputs. The kind of ANN can be divided into a feedforward neural network and cascade-forward neural network [30]. In this study, the multilayer cascade-forward ANN was applied as shown in Figure 6. The quantity of neurons in the first and second hidden layers is 6 and 6 respectively. In addition, the transfer function between the input layer and hidden layer and that between the hidden layer and output layer are the tansig function and purlin function respectively, which was defined as follows:

$$
\begin{gathered}
y^{2}=\operatorname{purlin}\left(\sum_{j=1}^{m} w_{j}^{2} \times \tan \operatorname{sig}\left(\sum_{i=1}^{n} w_{i, j}^{1} u_{i}^{1}+b_{j}^{1}\right)\right)+b^{2} \\
\tan \operatorname{sig}(\mathrm{x})=\frac{2}{1+e^{-2 x}}-1 \\
\operatorname{purlin}(\mathrm{x})=a \mathrm{x}+d
\end{gathered}
$$

where, superscript 1 and 2 stands for the coefficients from the first and second hidden layer. $w$ and $b$ mean the weight and biases for every neuron respectively. $a$ and $d$ is the constant coefficient. 


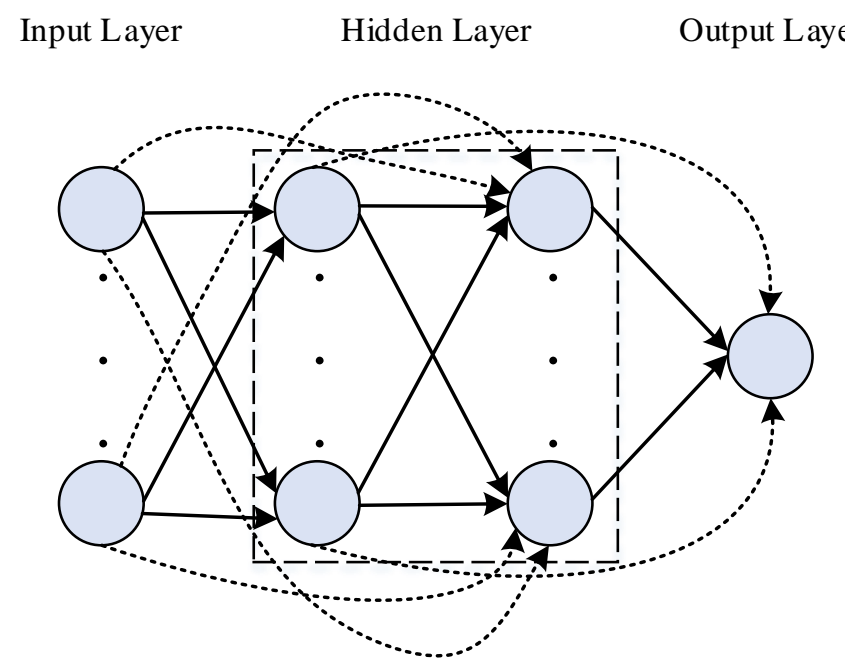

Figure 8. The structure of the cascade-forward multilayer neural network.

In order to prove that the cascade-forward two-layer ANN built function with high accuracy between the design variables and optimization objectives, the prediction accuracy of optimization objective compared with other common approximation models is shown in Table 7. Compared with other common approximate models, a two-layer ANN model can construct the function with highest accuracy between design variables and optimization objectives. The R-squared was set as the evaluation basis of the prediction accuracy and it is defined as follows:

$$
\begin{aligned}
S S E & =\sum_{i=1}^{n} \tau_{i}\left(y_{i}-\hat{y}_{i}\right)^{2} \\
S S T & =\sum_{i=1}^{n} \tau_{i}\left(y_{i}-\bar{y}_{i}\right)^{2} \\
R^{2} & =1-\frac{\sum(y-\hat{y})^{2}}{\sum(y-\bar{y})^{2}}
\end{aligned}
$$

where SSE, SST and $R^{2}$ mean the sum of squares due to the error, total sum of squares and coefficient of determination, respectively. $y, \hat{y}$ and $\bar{y}$ stand for the outputs of the sample points, predicted outputs by approximation models and average outputs for sample points.

Table 7. The accuracy of optimization objective predicted by different approximation models.

\begin{tabular}{ccccc}
\hline Approximation Model & $\boldsymbol{R}^{2}$ of $\boldsymbol{\eta}_{1} / \%$ & $\boldsymbol{R}^{2}$ of $\boldsymbol{\eta}_{2} / \%$ & $\boldsymbol{R}^{2}$ of $\boldsymbol{H}_{1} / \mathbf{m}$ & $\boldsymbol{R}^{2}$ of $\boldsymbol{H}_{2} / \mathbf{m}$ \\
\hline Two-layer ANN Model & 0.982 & 0.990 & 0.993 & 0.996 \\
RSM & 0.913 & 0.962 & 0.970 & 0.994 \\
Kriging Model & 0.792 & 0.953 & 0.938 & 0.948 \\
RBF Model & 0.905 & 0.983 & 0.972 & 0.993 \\
\hline
\end{tabular}




\subsection{NSGA for Multiobjective Optimization}

The non-dominated sorting genetic algorithm with the elitism algorithm (NSGA-II) was invoked from the genetic algorithm for multiobjective optimization. The advantage of this optimization algorithm is that the elitism sorting algorithm can reduce computational complexity. NSGA-II [31] is one of the popular multiobjective evolutionary algorithms. In this study, the objective function values were calculated by using two-layer ANN models. The size of the population and total number of the generation are 300 and 100, respectively. So, the NSGA-II was used to solve these approximate models. There were 300 Pareto-optimal solutions for the three optimization objectives as shown in Figure 9.

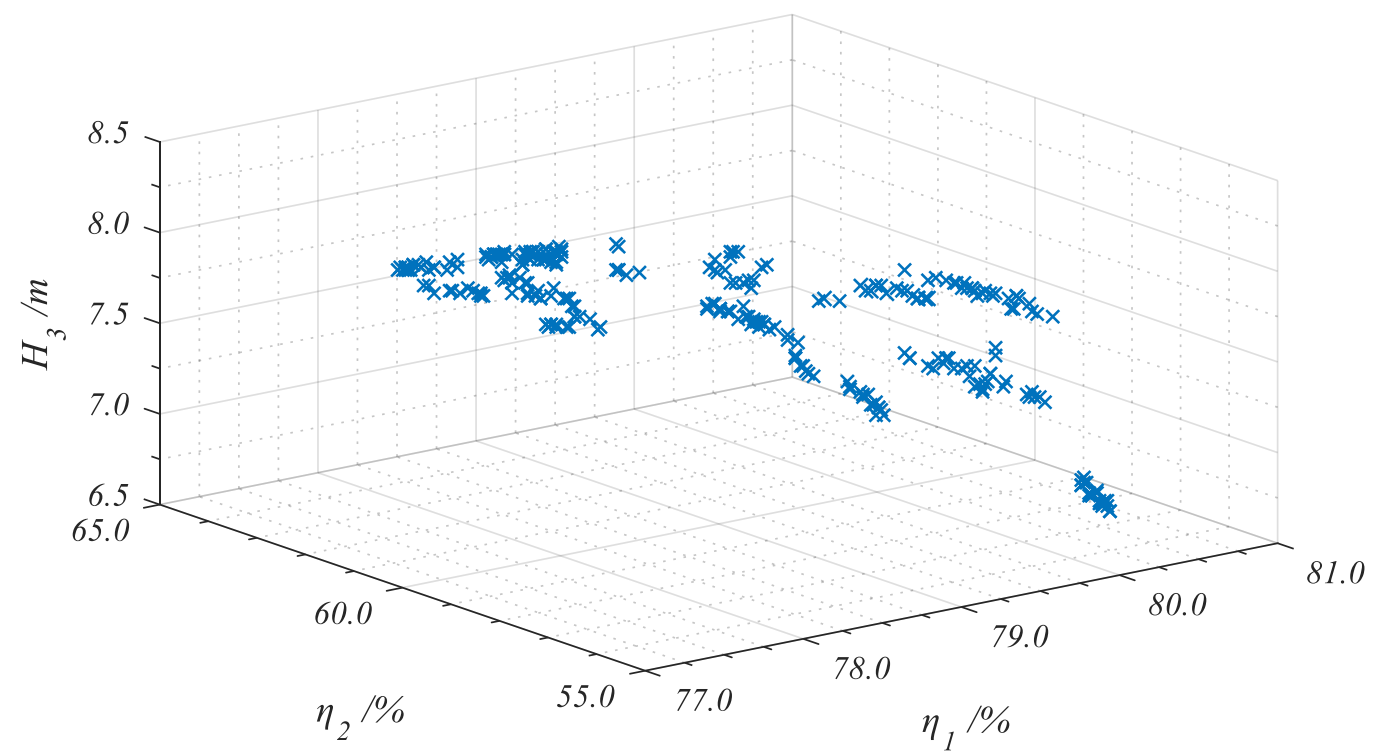

Figure 9. Pareto-optimal solutions in 3D function-space.

The Pareto-optimal solution is a set of solutions that are not inferior to all other feasible solutions. Generally it refers to improving the objective functions of a multiobjective problem while degrading some other objectives values. This is also referred to as non-dominated [32]. The optimized solutions were selected according to design requirements. In order to illustrate the relationship between the optimization objectives in detail, the Pareto-optimal solutions in 2D space was shown in Figure 10. Figure 10a shows the trade-off among Pareto-optimal solutions in the $\eta_{1}-H_{3}$ space, where $\eta_{1}$ increased with $\mathrm{H}_{3}$ decreasing. Additionally, Figure 10b shows a trade-off among Pareto-optimal solutions in $\eta_{1}-\eta_{2}$ space, where $\eta_{1}$ increased with $\eta_{2}$ decreasing. However, Figure 10c shows a weak relationship among Pareto-optimal solutions in $\eta_{2}-H_{3}$ space. $\eta_{1}, \eta_{2}$ and $H_{3}$ cannot be improved at the same time, but the sum of $\eta_{1}$ and $\eta_{2}$ can be improved after optimizing the pump and maintaining a certain lift. One representative Pareto-optimal solution was chosen based on Equations (26) and (27). The $\eta_{1}, \eta_{2}$ and $H_{3}$ predicted by NSGA-II were $78.13 \%, 61.01 \%$ and $8.07 \mathrm{~m}$. This representative Pareto-optimal solution was considered as the finally optimization design after a minor adjustment of the design variables based on experience.

$$
\begin{gathered}
\text { Maximum : } H_{3} \\
\text { Constraint : }\left\{\begin{array}{l}
\eta_{1}>78 \\
\eta_{2}>61
\end{array}\right.
\end{gathered}
$$




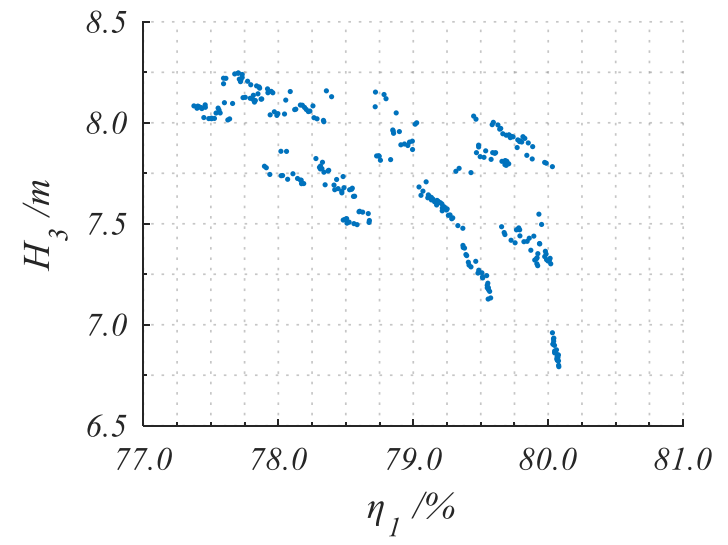

(a)

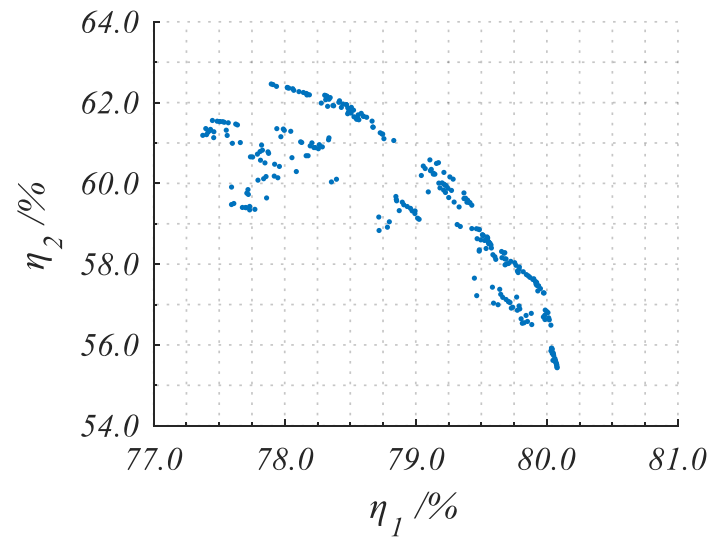

(b)

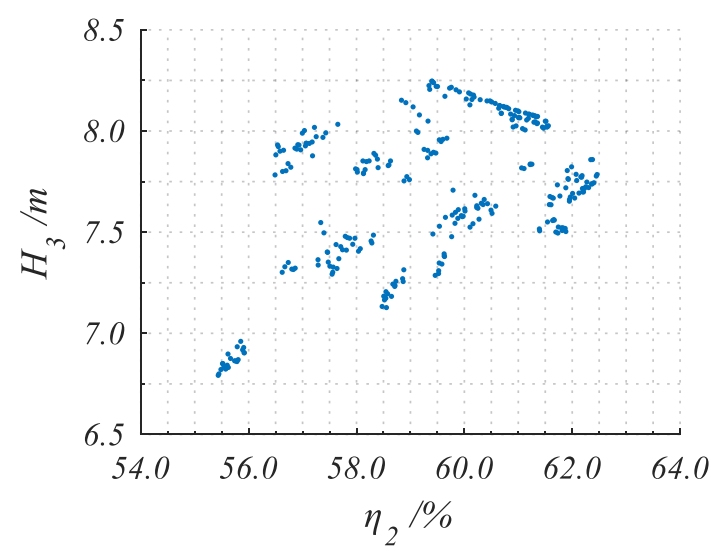

(c)

Figure 10. Pareto-optimal solutions in 2D function-space: (a) $\eta_{1}-H_{3 \prime}$, (b) $\eta_{1}-\eta_{2}$ and (c) $\eta_{2}-H_{3}$.

\section{Results and Analysis}

\subsection{Comparison of Pump Performance}

Figure 11 shows the comparison of pump efficiency and head between the original design and optimized design. Under the forward condition, the tendency of performance curves for the optimized design was similar with that for the original design, and the high efficiency zone was obtained under $Q=[0.36,0.4]\left(\mathrm{m}^{3} / \mathrm{s}\right)$. The pump performance under the forward condition and reverse condition could not be improved at the same time. In order to take $\eta_{2}$ and $H_{2}$ into consideration during the optimization design, forward pump performance under all flowrates slightly dropped after optimization. The $\eta_{1}$ and $H_{1}$ of the optimized design were $77.61 \%$ and $4.21 \mathrm{~m}$. However, under the reverse condition, the improvement of pump efficiency and the pump head were so obvious and the high efficiency range was also widened. The $\eta_{2}$ and $H_{2}$ of the optimized design were $61.58 \%$ and $3.8 \mathrm{~m}$. 


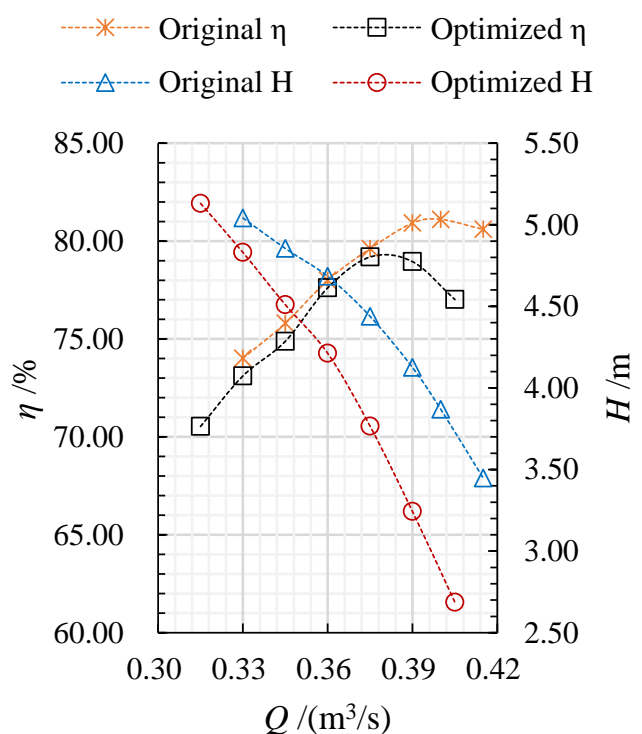

(a)

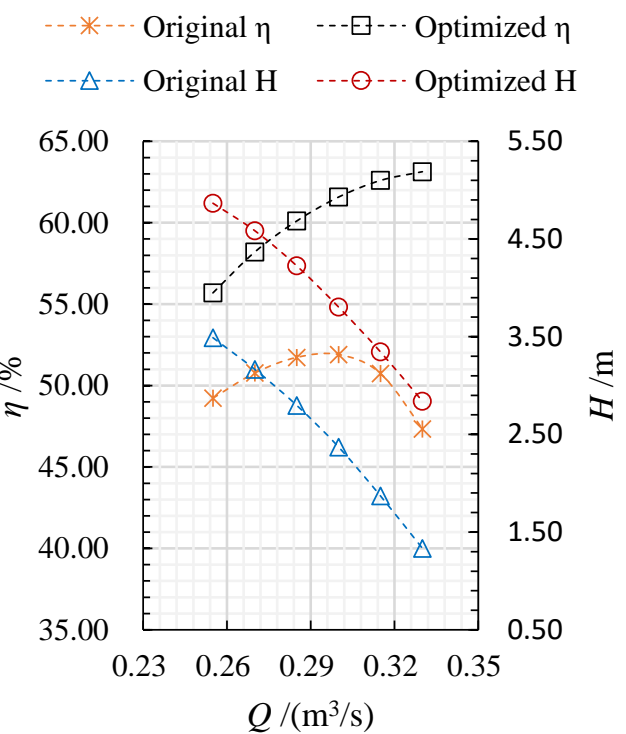

(b)

Figure 11. Comparison of pump performance between the original and optimized design: (a) under the forward condition and (b) under the reverse condition.

\subsection{Analysis of Inner Flow}

Figure 12 shows the turbo surface of the impeller with different span, and the span was defined as Equation (28).

$$
\text { Span }=\left(r-r_{h}\right) /\left(r_{t}-r_{h}\right)
$$

where, $r$ is the calculated ring radius, $r_{h}$ is the hub radius and $r_{t}$ is the radius of the impeller rim.

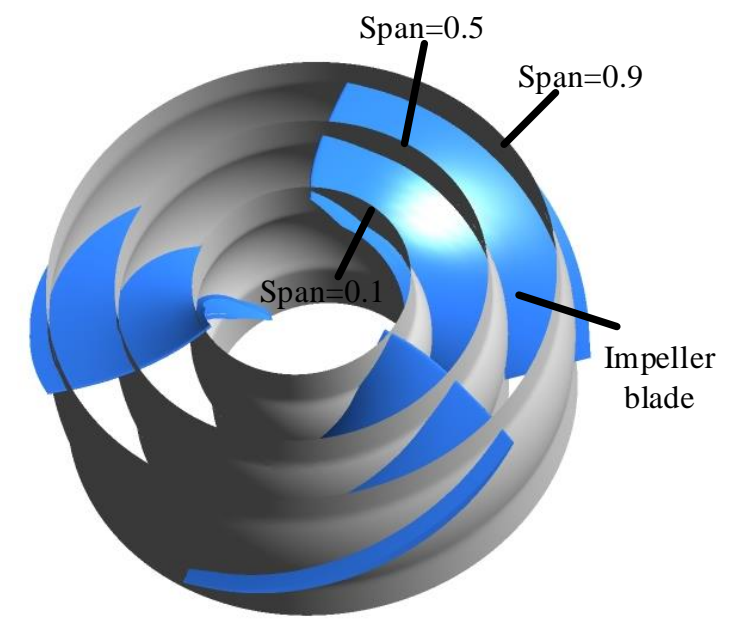

Figure 12. The turbo surface with different spans.

Figure 13 shows the velocity axial distribution in the expansion diagram of the turbo surface of the impeller with span $=0.5$, under the forward design flow rate. In the expansion diagram, the vertical axis was parallel to the impeller rotation axis and the horizontal axis was parallel to the circumferential direction of the impeller. The Ps and Ss stands for the pressure side and suction side. In both the original and optimized design, the high velocity was observed near the suction side and the low velocity near the leadging edge due to the presence of the stagnation point. After optimization, the velocity increased at the pressure side and appeared to be declining near the suction side. 
Low velocity near leading edge

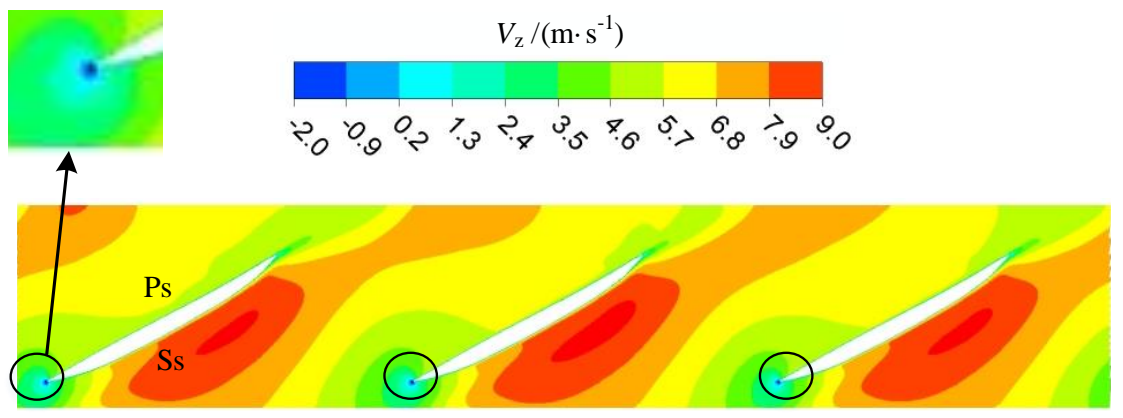

Original design

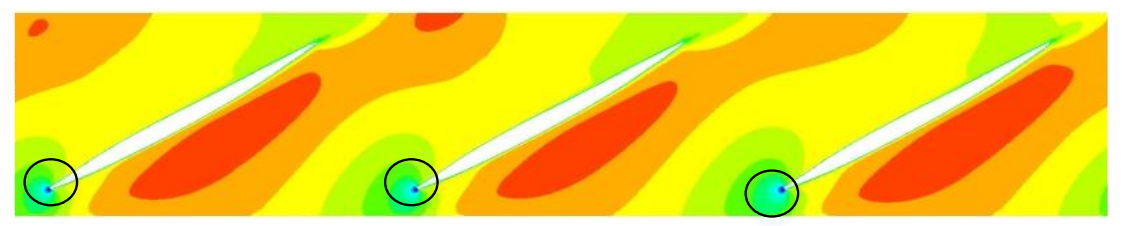

Optimized design

Figure 13. Comparison of the velocity axial distribution in the impeller between the original design and optimized design under the forward design flow rate $($ span $=0.5)$.

Figure 14a shows the axial velocity distribution in the impeller outlet under the forward design flow rate. The trend of the velocity curve in the optimized design was similar with that of the original design. The low axial velocity could be seen near span $=0$ and span $=1.0$ due to the viscous effects caused by the friction between the fluid and wall. In the middle interval, the axial velocity increased slowly with increasing span. In addition, when span $<0.6$, the axial velocity in the original design was smaller than that of the optimized design, but when span $>0.6$, the axial velocity in the original design was larger than that in the optimized design. Figure 14b shows the circulation distribution in the impeller outlet. The circulation was defined as Equation (29). The tendency of the velocity curve in the optimized design was also similar with that in the original design. The circulation rose slowly with span increasing firstly and then it increased rapidly with span increasing. Furthermore, the circulation in the optimized design was smaller than that of the original design, which would cause the pump head to decline after optimization.

$$
C_{\mathrm{u}}=\int_{L} \boldsymbol{v}_{u} d \boldsymbol{l}
$$

where, $v_{u}$ is the absolute circumferential velocity and $d l$ is the unit length of the ring.

Figure 15 shows the axial velocity distribution in the expansion diagram of the turbo surface with span $=0.5$ under the forward design flow rate. As shown in the figure, both in the original design and optimized design, the difference of the flow structure near each diffuser blade was so obvious. The high velocity could be found near some diffuser blades and the obvious low velocity was observed near the rest diffuser blades, which could result in hydraulic losses. 


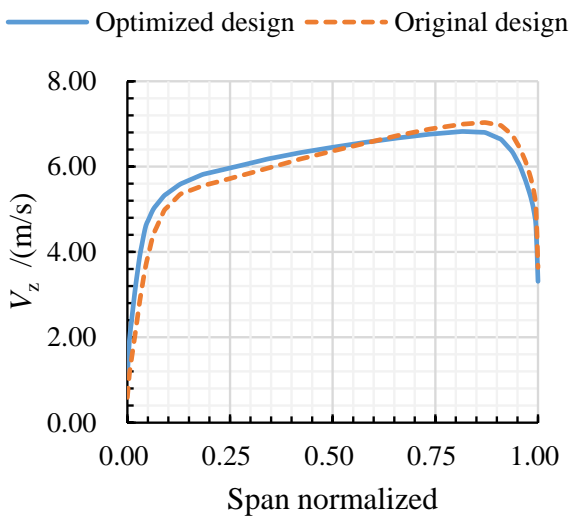

(a)

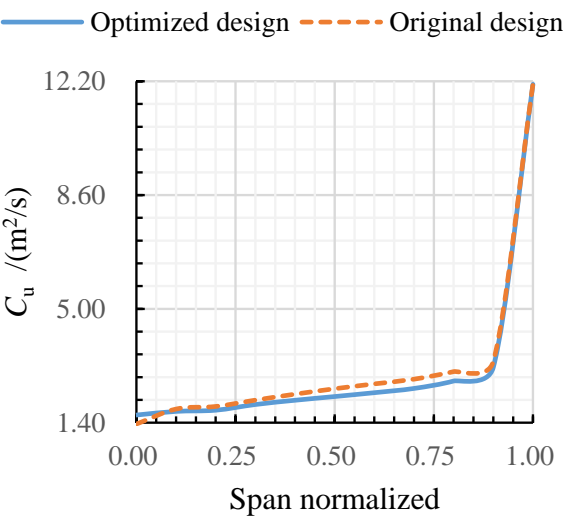

(b)

Figure 14. Comparison in the impeller outlet between the original and optimized design for the (a) velocity axial distribution and (b) circulation distribution (under the forward design flow rate).

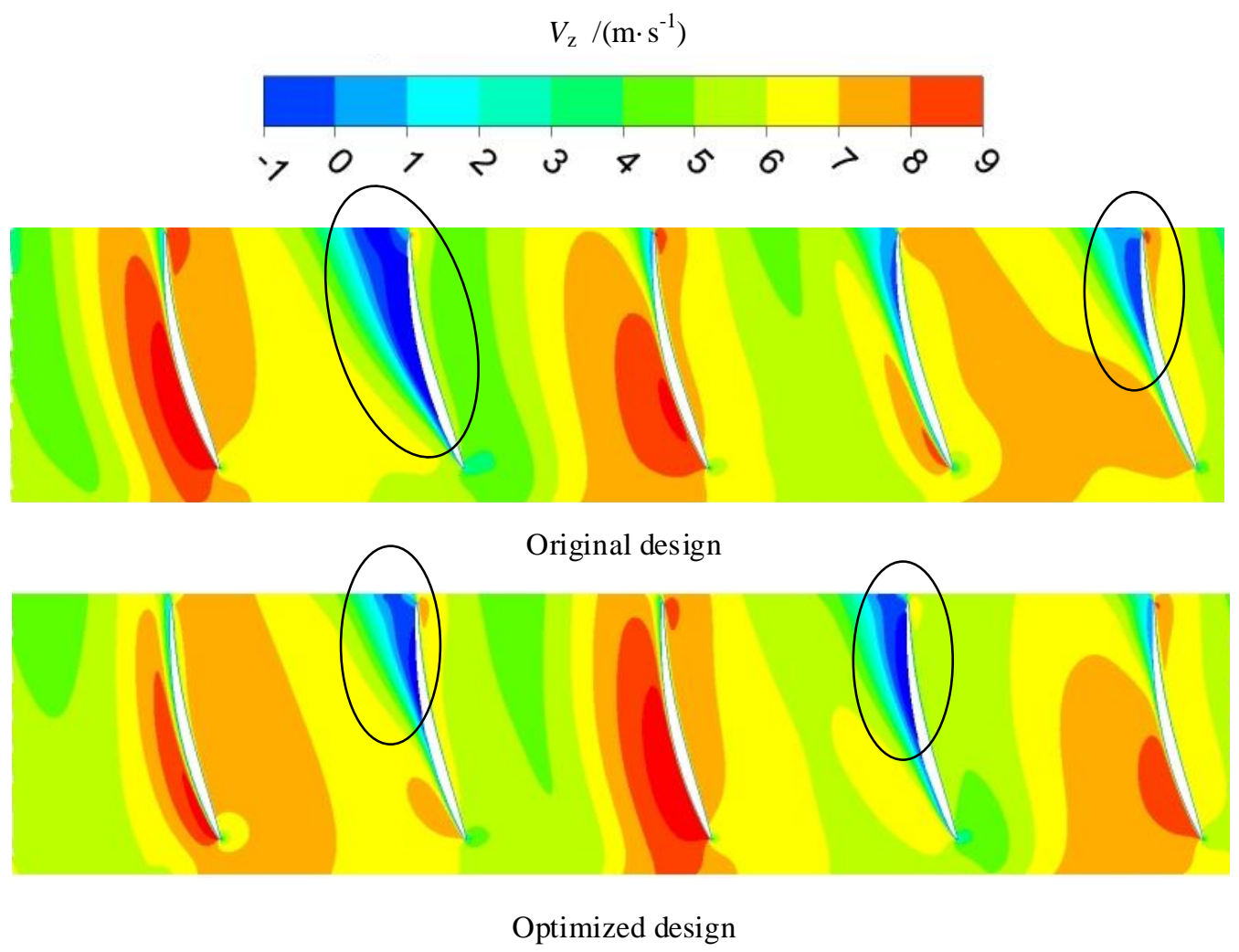

Figure 15. Comparison of the velocity axial distribution in the diffuser between the original and optimized design under the forward design flow rate $(\operatorname{span}=0.5)$.

Figure 16 is the distribution of the axial velocity in the expansion diagram of the turbo surface with span $=0.5$ under the reverse design flow rate. These two designs show a similar flow structure with weak velocity near the leading edge, which was due to the presence of the stagnation point of the leading edge, as well as the high velocity near the suction side. In the optimized design, the area of the high velocity near the suction side became larger, and the velocity near the pressure side became lower. The velocity gradient near the pressure side in the original design was larger than that in the optimized design. 
Low velocity near leading edge

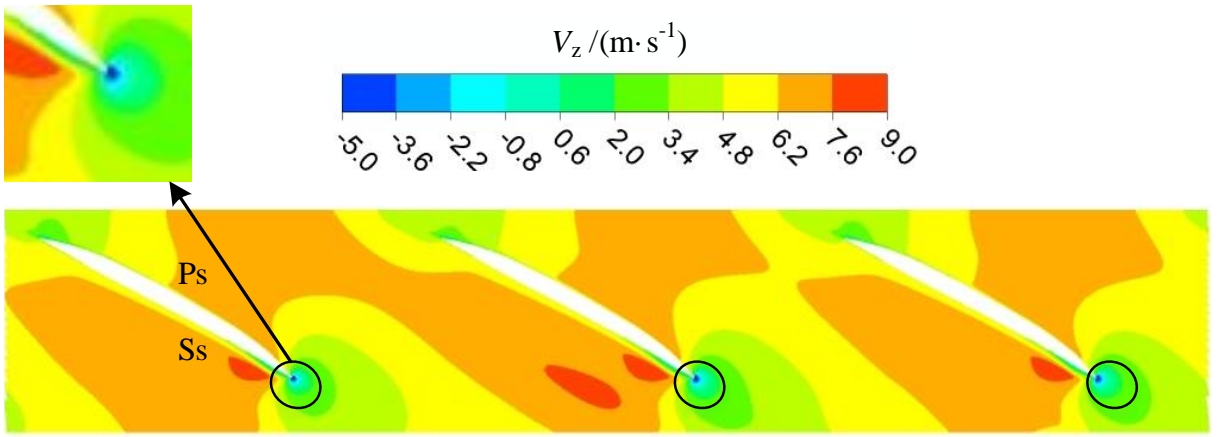

Original design

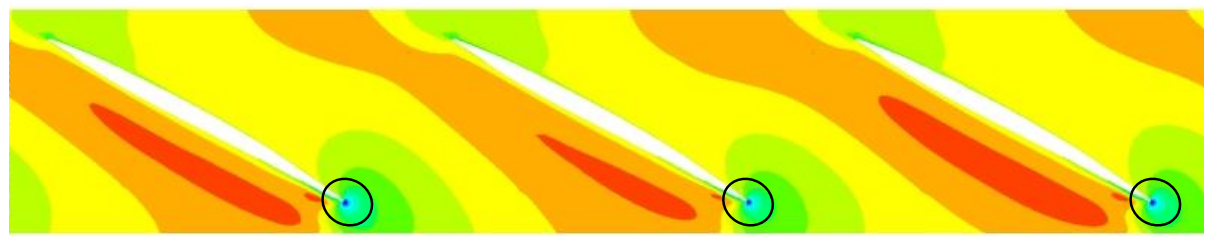

Optimized design

Figure 16. Comparison of the velocity distribution in the impeller between the original design and optimized design under the reverse design flow rate ( $\operatorname{span}=0.5)$.

Figure 17 shows the comparison of the velocity in the impeller outlet between the original and optimized design. As shown in Figure 17a, the original impeller had a non-uniform axial velocity distribution, and it first increased, then decreased with increasing span in the middle interval. The axial velocity of the optimized design remained almost stable in the middle interval, which meant that the impeller outflow was much more stable. As shown in Figure 17b, the tendency of the circulation curve for the optimized design was similar with that for the original design. The circulation initially rose slowly and later became rapid with increasing span. The optimized impeller outlet however had higher circulation when span $>0.25$ and it could result in a higher pump head compared with the original design.

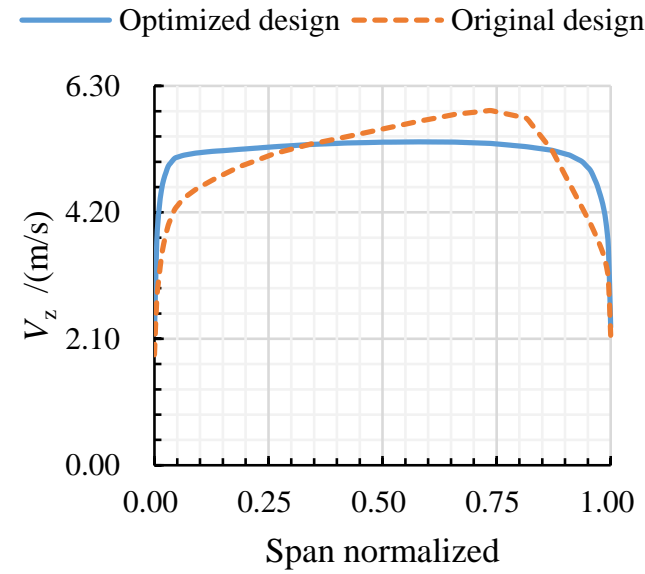

(a)

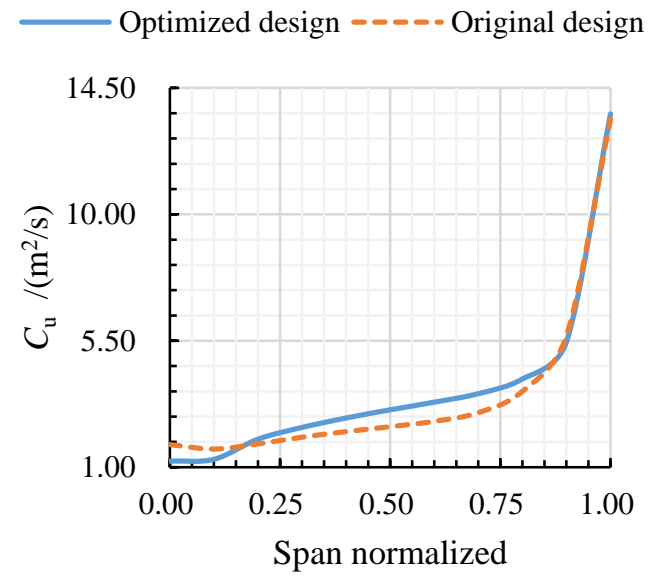

(b)

Figure 17. Comparison in the impeller outlet between the original and optimized design for the (a) axial velocity distribution and (b) circumferential velocity distribution (under the reverse design flow rate). 


\subsection{Validation of the Optimization Results}

In order to prove the validity of the optimization design and accuracy of the numerical simulation, the external characteristic test for the optimized design was carried out. Figure 18 shows the vertical bench test of the axial-flow pump and the flow rate was regulated by the gate valve. The main technical parameters of the measurement equipment were shown in Table 8. Due to the structure of the test bench, the rotation speed of $1350 \mathrm{r} / \mathrm{min}$ was used for the external characteristic test. Then the experiment data was transformed by the equivalent efficiency formula to compare with the numerical simulation as shown in Figure 19. The performance curves from the numerical simulation had good agreement with experiment data, and the deviation of $\eta_{1}$ and $\eta_{2}$ were about $1.23 \%$ and $3.56 \%$. In addition, the main design parameters of this optimized reversible axial-flow pump is shown in Table 9. The results shows that the reverse pump performance was improved actually, at the same time of maintaining the forward pump performance.

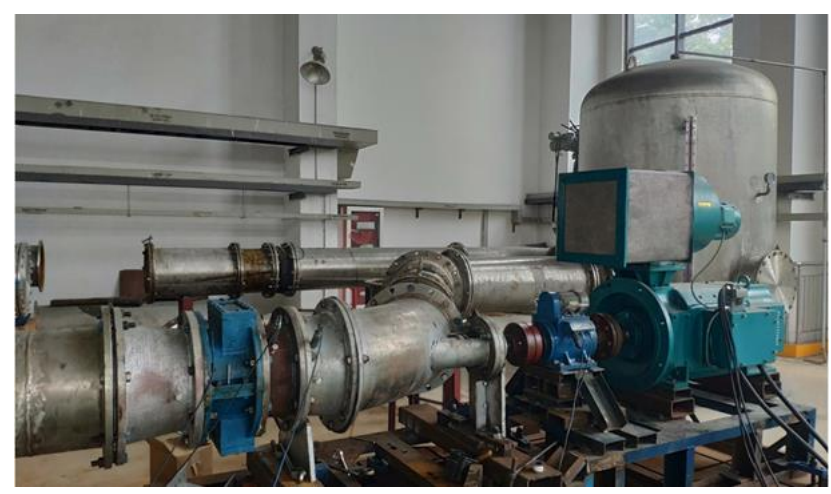

Figure 18. The experiment bench of the axial-flow pump.

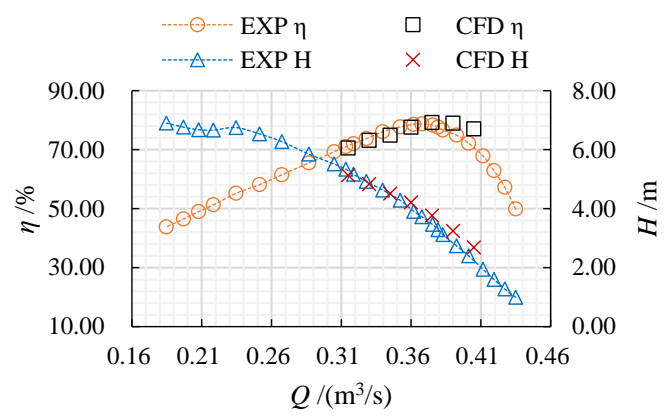

(a)

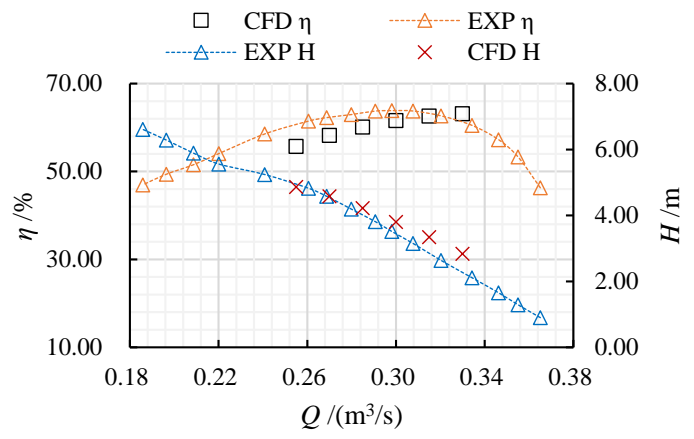

(b)

Figure 19. The comparison of pump performance between the simulated data and experiment data under the (a) forward operation and (b) reverse operation. 
Table 8. Main technical parameters of measurement equipment.

\begin{tabular}{ccccc}
\hline $\begin{array}{c}\text { Measurement } \\
\text { Items }\end{array}$ & Equipment Name & Instrument Model & $\begin{array}{c}\text { Measurement } \\
\text { Range }\end{array}$ & $\begin{array}{c}\text { Measurement } \\
\text { Accuracy }\end{array}$ \\
\hline Flow rate & $\begin{array}{c}\text { Intelligent electromagnetic } \\
\text { flowmeter } \\
\text { Intelligent differential } \\
\text { pressure transmitter }\end{array}$ & OPTIFLUX2000F & $0-1800 \mathrm{~m}^{3} / \mathrm{h}$ & $< \pm 0.2 \%$ \\
$\begin{array}{c}\text { Head } \\
\text { Torque } \\
\text { Rotation speed }\end{array}$ & $\begin{array}{c}\text { EJA } \\
\text { sensor }\end{array}$ & JCL1 & $0-10 \mathrm{~m}$ & $< \pm 0.1 \%$ \\
\hline
\end{tabular}

Table 9. The main design parameters of the optimized reversible axial-flow pump.

\begin{tabular}{cc}
\hline Design Parameters & Value \\
\hline Forward rated flowrate $\left(\mathrm{m}^{3} / \mathrm{s}\right)$ & 0.368 \\
Efficiency under forward rated condition $(\%)$ & 78.73 \\
Head under forward rated condition $(\mathrm{m})$ & 3.72 \\
Reverse rated flowrate $\left(\mathrm{m}^{3} / \mathrm{s}\right)$ & 0.298 \\
Efficiency under reverse rated condition $(\%)$ & 63.85 \\
Head under reverse rated condition $(\mathrm{m})$ & 3.51 \\
Rotation speed $(\mathrm{rev} / \mathrm{min})$ & 1450 \\
\hline
\end{tabular}

\section{Conclusions}

In this paper, a multiobjective optimization was performed to improve the total pump performance under the forward and reverse design condition. Some conclusions could be drawn.

(1) $\quad \alpha_{5}$ and $\varphi_{1}$ had the most important effect on the pump performance, according to the orthogonal experimental design. $L_{1}, L_{5}, \theta_{1}, \theta_{5}, \alpha_{5}$ and $\varphi_{1}$ were set as the final design variables for the combination optimization of the impeller and diffuser.

(2) Compared with other approximate models, the two-layer ANN could construct the functions with highest accuracy between the design variables and optimization objectives, based on 120 groups of sample points generated from LHS.

(3) 300 Pareto-optimal solutions were obtained by NSGA and one representative Pareto-optimal solution was selected as the basis of the optimized design. The $\eta_{1}$ and $H_{1}$ of the optimized design decreased by $0.50 \%$ and $10.06 \%$, respectively, compared with the original design. The axial velocity gradient near the suction side of the optimized diffuser was larger than that of the original diffuser. The circulation of the impeller outlet had decreased after optimization. However, the $\eta_{2}$ and $\mathrm{H}_{2}$ of the optimized design increased by $18.62 \%$ and $60.40 \%$, compared with the original design. In addition, the curve slope of the axial velocity and circulation in the impeller outlet of the optimized design decreased and increased after optimization.

(4) The external characteristic test for the optimized design was completed to prove the validity of this optimization method. In addition, the test highest efficiency values under the forward condition and reverse condition were $78.73 \%$ and $63.85 \%$ respectively, which could meet engineering needs.

The design method in this paper could be used as a reliable and high time-efficient methodology to design the reversible axial-flow pump based on a one-way axial-flow pump.

Author Contributions: Conceptualization, F.M.; Data curation, F.M.; Methodology, F.M.; Project administration, Y.L., S.Y. and W.W.; Supervision, Y.L.; Validation, F.M.; Writing-original draft, F.M.; Writing-review \& editing, Y.Z. and M.K.O. All authors have read and agreed to the published version of the manuscript.

Funding: This research was funded by National Key Research and Development Plan (Grant No.2018YFB0606103), Science and Technology Plan of Wuhan (Grant No.2018060403011350). Primary Research and Development Plan of Jiangsu Province (Grant No. BE2019009-1). Natural Science Foundation of Jiangsu University (Grant No. BK20190851), China Postdoctoral Science Foundation funded project (Grant No. 2019M651736).

Acknowledgments: The author sincerely thanks the 4Cpump researcher group for its help.

Conflicts of Interest: The authors declare no conflict of interest. 


\section{Nomenclature}

\begin{tabular}{ll}
$Q\left(\mathrm{~m}^{3} / \mathrm{s}\right)$ & Flow rate \\
$H_{1}(\mathrm{~m})$ & Head under forward design condition \\
$H_{2}(\mathrm{~m})$ & Head under reverse design condition \\
$H_{3}(\mathrm{~m})$ & Sum of $H_{1}$ and $H_{2}$ \\
$\eta_{1}(\%)$ & Efficiency under forward design condition \\
$\eta_{2}(\%)$ & Efficiency under forward design condition \\
$N(\mathrm{r} / \mathrm{min})$ & Rotating speed \\
$\theta\left(^{\circ}\right)$ & Blade angle of impeller \\
$L(\mathrm{~mm})$ & Chord length of impeller \\
$\varnothing\left(^{\circ}\right)$ & Included angle between camber line and chord \\
$\sigma\left(^{\circ}\right)$ & theoretical lift coefficient \\
$\lambda\left(^{\circ}\right)$ & Outlet angle of skeleton line \\
$\varepsilon\left(^{\circ}\right)$ & Inlet angle of skeleton line \\
$\alpha\left(^{\circ}\right)$ & Inlet angle of diffuser \\
$\beta\left(^{\circ}\right)$ & Outlet angle of diffuser \\
$\varphi\left(^{\circ}\right)$ & Wrap angle of diffuser \\
$C_{\mathrm{u}}\left(\mathrm{m}^{2} / \mathrm{s}\right)$ & Circulation \\
$V_{\mathrm{Z}}(\mathrm{m} / \mathrm{s})$ & Axial velocity \\
$\mathrm{Abbreviations}$ & \\
$3 \mathrm{D}$ & Three-Dimensional \\
$\mathrm{RANS}$ & Reynolds-Averaged Navier-Stokes \\
$\mathrm{LHS}_{\mathrm{ANN}}^{\mathrm{N}}$ & Latin Hypercube Sampling \\
$\mathrm{NSGA}$ & Artificial Neural Network \\
$\mathrm{CFD}$ & Non-domination based Genetic Algorithm \\
$\mathrm{DOE}$ & Computational Fluid Dynamics \\
$\mathrm{RSM}$ & Design of Experiment \\
$\mathrm{EXP}$ & Response Surface Model \\
& Experiment \\
\hline
\end{tabular}

\section{References}

1. Shi, L.; Liu, X.; Tang, F.; Yao, Y. Design optimization and experimental analysis of bidirectional shaft tubular pump device. Trans. Chin. Soc. Agric. Mach. 2016, 47, 85-91. (In Chinese)

2. Xie, R.; Wu, Z.; He, Y. Optimization Research on Passage of Bidirectional Shaft Tubular Pump. Trans. Chin. Soc. Agric. Mach. 2015, 46, 68-74. (In Chinese)

3. Xia, Y.; Tang, F.; Shi, L.; Xie, C.; Zhang, W. Numerical Simulation and Experimental Analysis of Bidirectional Shaft Tubular Pump Device. China Rural Water Hydropower 2017, 7, 34. (In Chinese)

4. Feng, J.; Luo, X.; Guo, P.; Wu, G. Influence of tip clearance on pressure fluctuations in an axial flow pump. J. Mechan. Sci. Technol. 2016, 30, 1603-1610. [CrossRef]

5. Pei, J.; Yuan, S.; Benra, F.K.; Dohmen, H.J. Numerical prediction of unsteady pressure field within the whole flow passage of a radial single-blade pump. J. Fluids Eng. 2012, 134, 101103. [CrossRef]

6. Zhang, D.; Shi, L.; Zhao, R.; Shi, W.; Pan, Q.; van Esch, B.B. Study on unsteady tip leakage vortex cavitation in an axial-flow pump using an improved filter-based model. J. Mech. Sci. Technol. 2017, 31, 659-667. [CrossRef]

7. Dong, L.; Shang, H.; Zhao, Y.; Liu, H.; Dai, C.; Wang, Y. Study on Unstable Characteristics of Centrifugal Pump under Different Cavitation Stages. J. Therm. Sci. 2019, 28, 608-620. [CrossRef]

8. Yang, B.; Li, B.; Chen, H.; Liu, Z. Entropy production analysis for the clocking effect between inducer and impeller in a high-speed centrifugal pump. Proc. Inst. Mech. Eng. Part C J. Mech. Eng. Sci. 2019, 233, 5302-5315. [CrossRef]

9. Zhang, F.; Yuan, S.; Wei, X.; Chen, K. Study on Flow Loss Characteristics of Side Channel Pump Based on Entropy Production. J. Mechan. Eng. 2018, 54, 137-144. (In Chinese) [CrossRef]

10. Yun, L.; Rongsheng, Z.; Dezhong, W.; Junlian, Y.; Tianbin, L. Numerical and experimental investigation on the diffuser optimization of a reactor coolant pump with orthogonal test approach. J. Mech. Sci. Technol. 2016, 30, 4941-4948. [CrossRef] 
11. Liu, H.; Chen, X.; Wang, K.; Tan, M.; Zhou, X. Multi-condition optimization and experimental study of impeller blades in a mixed-flow pump. Adv. Mech. Eng. 2016, 8. [CrossRef]

12. Pei, J.; Gan, X.; Wang, W.; Yuan, S.; Tang, Y. Multi-objective shape optimization on the inlet pipe of a vertical inline pump. J. Fluids Eng. 2019, 141, 061108. [CrossRef]

13. Miao, F.; Park, H.S.; Kim, C.; Ahn, S. Swarm intelligence based on modified PSO algorithm for the optimization of axial-flow pump impeller. J. Mech. Sci. Technol. 2015, 29, 4867-4876. [CrossRef]

14. Shim, H.S.; Afzal, A.; Kim, K.Y.; Jeong, H.S. Three-objective optimization of a centrifugal pump with double volute to minimize radial thrust at off-design conditions. Proc. Inst. Mech. Eng. Part A J. Power Energy 2016, 230, 598-615. [CrossRef]

15. Shim, H.S.; Kim, K.Y.; Choi, Y.S. Three-objective optimization of a centrifugal pump to reduce flow recirculation and cavitation. J. Fluids Eng. 2018, 140, 091202. [CrossRef]

16. Wang, W.; Yuan, S.; Pei, J.; Zhang, J. Optimization of the diffuser in a centrifugal pump by combining response surface method with multi-island genetic algorithm. Proc. Inst. Mech. Eng. Part E J. Process Mech. Eng. 2017, 231, 191-201. [CrossRef]

17. Wang, W.; Pei, J.; Yuan, S.; Zhang, J.; Yuan, J.; Xu, C. Application of different surrogate models on the optimization of centrifugal pump. J. Mech. Sci. Technol. 2016, 30, 567-574. [CrossRef]

18. Ma, P.; Wang, J.; Li, H. Numerical analysis of pressure pulsation for a bidirectional pump under positive and reverse operation. Adv. Mech. Eng. 2014, 6, 730280. [CrossRef]

19. Ma, P.F.; Wang, J.; Wang, H.F. Investigation of performances and flow characteristics of two bi-directional pumps with different airfoil blades. Sci. China Technol. Sci. 2018, 61, 1588-1599. [CrossRef]

20. Jung, I.S.; Jung, W.H.; Baek, S.H.; Kang, S. Shape optimization of impeller blades for a bidirectional axial flow pump using polynomial surrogate model. World Acad. Sci. Eng. Technol. 2012, 66, 718-724.

21. Morris, M.D. Factorial sampling plans for preliminary computational experiments. Technometrics 1991, 33, 161-174. [CrossRef]

22. Menter, F.; Ferreira, J.C.; Esch, T.; Konno, B.; Germany, A.C. The SST Turbulence Model with Improved Wall Treatment for Heat Transfer Predictions in Gas Turbines. In Proceedings of the International Gas Turbine Congress 2003, Tokyo, Japan, 2-7 November 2003.

23. Campolongo, F.; Cariboni, J.; Saltelli, A. An effective screening design for sensitivity analysis of large models. Environ. Model Softw. 2007, 22, 1509-1518. [CrossRef]

24. McKay, M.D.; Beckman, R.J.; Conover, W.J. Comparison of three methods for selecting values of input variables in the analysis of output from a computer code. Technometrics 1979, 21, 239-245.

25. Kim, J.H.; Kim, K.Y. Analysis and optimization of a vaned diffuser in a mixed flow pump to improve hydrodynamic performance. J. Fluids Eng. 2012, 134, 071104. [CrossRef]

26. Zhu, B.; Wang, X.; Tan, L.; Zhou, D.; Zhao, Y.; Gao, S. Optimization design of a reversible pump-turbine runner with high efficiency and stability. Renew. Energy 2015, 81, 366-376. [CrossRef]

27. Simpson, T.W.; Mauery, T.M.; Korte, J.J.; Mistree, F. Kriging models for global approximation in simulation-based multidisciplinary design optimization. AIAA J. 2001, 39, 2233-2241. [CrossRef]

28. Heo, M.W.; Ma, S.B.; Shim, H.S.; Kim, K.Y. High-efficiency design optimization of a centrifugal pump. J. Mechan. Sci. Technol. 2016, 30, 3917-3927. [CrossRef]

29. Rossi, M.; Renzi, M. A general methodology for performance prediction of pumps-as-turbines using Artificial Neural Networks. Renew. Energy 2018, 128, 265-274. [CrossRef]

30. Pei, J.; Wang, W.; Osman, M.K.; Gan, X. Multiparameter optimization for the nonlinear performance improvement of centrifugal pumps using a multilayer neural network. J. Mech. Sci. Technol. 2019, 33, 2681-2691. [CrossRef]

31. Deb, K.; Pratap, A.; Agarwal, S.; Meyarivan, T.A. A fast and elitist multiobjective genetic algorithm: NSGA-II. IEEE Trans. Evol. Comput. 2002, 6, 182-197. [CrossRef]

32. Zhang, J.; Zhu, H.; Yang, C.; Li, Y.; Wei, H. Multi-objective shape optimization of helico-axial multiphase pump impeller based on NSGA-II and ANN. Energy Convers. Manag. 2011, 52, 538-546. [CrossRef] 